\title{
The speed of Arnold diffusion
}

\author{
C. Efthymiopoulos ${ }^{1}$, M. Harsoula ${ }^{1}$ \\ ${ }^{a}$ Research Center for Astronomy and Applied Mathematics, Academy of Athens
}

\begin{abstract}
A detailed numerical study is presented of the slow diffusion (Arnold diffusion) taking place around resonance crossings in nearly integrable Hamiltonian systems of three degrees of freedom in the so-called 'Nekhoroshev regime'. The aim is to construct estimates regarding the speed of diffusion based on the numerical values of a truncated form of the so-called remainder of a normalized Hamiltonian function, and to compare them with the outcomes of direct numerical experiments using ensembles of orbits. In this comparison we examine, one by one, the main steps of the so-called analytic and geometric parts of the Nekhoroshev theorem. Thus: i) we review and implement an algorithm [20] for Hamiltonian normalization in multiply resonant domains which is implemented as a computer program making calculations up to a high normalization order. ii) We compute the dependence of the optimal normalization order on the small parameter $\epsilon$ in a specific model and compare the result with theoretical estimates on this dependence. iii) We examine in detail the consequences of assuming simple convexity conditions for the unperturbed Hamiltonian on the geometry of the resonances and on the phase space structure around resonance crossings. iv) We discuss the dynamical mechanisms by which the remainder of the optimal Hamiltonian normal form drives the diffusion process. Through these steps, we are led to two main results: i) We construct in our concrete example a convenient set of variables, proposed first by Benettin and Gallavotti [4], in which the phenomenon of Arnold diffusion in doubly resonant domains can be clearly visualized. ii) We determine, by numerical fitting of our data the dependence of the local diffusion coefficient $D$ on the size $\left\|R_{\text {opt }}\right\|$ of the optimal remainder function, and we compare this with a heuristic argument based on the assumption of normal diffusion. We find a power law $D \propto\left\|R_{o p t}\right\|^{2(1+b)}$, where the constant $b$ has a small positive value depending also on the multiplicity of the resonance considered.
\end{abstract}

Keywords: Hamiltonian systems; Arnold diffusion; normal forms; Nekhoroshev theorem.

\section{Introduction}

The study of diffusion in nearly-integrable Hamiltonian dynamical systems of the form

$$
H(I, \phi)=H_{0}(I)+\epsilon H_{1}(I, \phi)
$$

where $(I, \phi)$ are n-dimensional action - angle variables and $\epsilon$ is a small parameter, constitutes a central problem in Hamiltonian dynamical systems theory, in view, in particular, of its multiple

\footnotetext{
Email addresses: cefthim@academyofathens.gr (C. Efthymiopoulos), mharsoul@academyof athens.gr (M. Harsoula) 
applications in physics and astronomy (see [44][14] for an introduction, the basic review paper [9], or [47] 11] [39] for recent advanced reviews emphasizing various aspects of this subject). It is a well established result that, if $n>2$, and $H$ satisfies appropriate convexity and analyticity conditions (see section 2 below), two distinct regimes characterize the laws of diffusion as a function of $\epsilon$ : for $\epsilon<\epsilon_{*}$, where $\epsilon_{*}$ is a threshold value, the onset of the so-called "Nekhoroshev regime' takes place [51] [3] [45] [54][48] [47]. In this case, the Nekhoroshev theorem provides an $O\left[\exp \left(-\left(\epsilon_{*} / \epsilon\right)^{c}\right)\right]$ upper bound for the speed of diffusion. The exponent $c$ depends on the number of degrees of freedom $n$, while its precise value in local domains of the action space depends also on the multiplicity of the resonance conditions holding in such domains (see e.g. [4][54][48]). Furthermore, the mechanism of diffusion caused by transition chains, as demonstrated in one special example by Arnold [2] (see also [56]), is conjectured to hold in more general systems of the form (1) (e.g. [46]; note, however, that no formal proof of this fact has been given to date). On the other hand, for $\epsilon>\epsilon_{*}$, the diffusion is driven mainly by the mechanism of resonance overlap [55][13] [9]. In this case, one expects a power-law dependence of the speed of diffusion on $\epsilon$ (see e.g. [9]; a power law is also found in the case of the so-called 'Fast Arnold diffusion' [10]).

The diffusion in weakly chaotic systems has been a subject also of extensive numerical studies over the last three decades (some indicative references are [36] [59] [37] [15] [58] [16] [25] [7]). A detailed study, however, of the very slow diffusion characterizing the 'Nekhoroshev regime' has become possible only in recent years. In this respect, we note in particular the series of instructive works [22] [38] [23] [33][35], where, using the so-called Fast Lyapunov Indicator (FLI; see [22]), a method was found to depict the resonant structure of the action space in models of three degrees of freedom, or 4D and 6D symplectic mappings being in the Nekhoroshev regime [22, 23, 35]. In [38], the mean-square spread in action space $\left\langle\Delta J^{2}>\right.$ was measured as a function of the time $t$ for orbits along the chaotic border of a simply-resonant domain (see section 2 for a precise definition). It was found that i) the local character of diffusion is normal, i.e. $\left\langle\Delta J^{2}\right\rangle \propto t$, and ii) the diffusion coefficient $D=<\Delta J^{2}>/ t$ decreases with $\epsilon$ faster than a power law. The exponential fit $D \propto \exp \left(-\left(\epsilon_{c} / \epsilon\right)^{0.28}\right.$ was given in a subsequent study [23]. The estimate obtained in [35], through interpolation over five orders of magnitude of the perturbation parameter, yields with with certainty the first digit of the exponent $0.2 \ldots$, but the errors in the interpolation make uncertain the second digit in both the above estimates. In [41], $D$ was measured as a function of the separatrix splitting $S$ of the asymptotic manifolds of simply unstable two-dimensional tori lying at the borders of simple resonances (see also [49][40]). The measurement of $S$ itself was based on employing the FLI. It was found that $D \propto S^{p}$, with $p=2.1$ and $p=2.56$ in two resonances of increasing order respectively. Finally, the laws of diffusion in systems violating one or more necessary conditions of the Nekhoroshev theorem were investigated in [34] [42], leading to a number of interesting results regarding the dynamical consequences of such violations.

The motivation for the present study stems primarily from the results reported in refs [38] [23] [33] [41], and it can be described as follows. The results obtained so far are very satisfactory from the numerical point of view. They require, however, computations involving large ensembles of orbits and integration times of the order of billions, or even trillions of periods. On the other hand, we can remark that, in principle, the analytical methods involved in the main theories of chaotic diffusion lend themselves also conveniently to getting quantitative predictions regarding the value of the diffusion coefficient, or the scaling laws of diffusion, in general, in the weakly chaotic regime. For such a goal, however, to be accomplished, it is required that one should be able to carry on expansions of certain quantities up to a very high order in the small parameter $\epsilon$ (usually with the aid of a computer). This fact is explicit in Nekhoroshev theory, where one 
needs to reach an expansion order high enough for the asymptotic behavior of the perturbation series to show up. This has been realized in studies seeking to determine the range (in the small parameter value) and/or the conditions of applicability of Nekhoroshev theory, or, finally, the domain of practical stability for motions in simple physical systems or models inspired mainly from Solar System dynamics (see e.g. [26][8] [48][28][18][19] [43][52][31]). These studies notwithstanding, the question of central interest in the present paper, namely how to obtain relevant quantitative estimates of the local value of the diffusion coefficient $D$ in resonant domains (of various multiplicities) of the action space via high order expansions of perturbation theory, remains, to our knowledge, largely unexplored.

Regarding now this last question, it should be noted that the formal analytical apparatus of Nekhoroshev theory, entailing the construction of a normal form in local domains covering the action space of systems of the form (1), aims to transform the original Hamiltonian into one in new canonical variables resuming the form $H_{\text {transformed }}=Z+R$, where $Z$, the normal form, corresponds to a simple dynamics, while $R$, the remainder, induces a perturbation to this dynamics. The so-called 'geometric part' of Nekhoroshev the theorem ensures that, despite allowing in general for chaotic motions, the flow under a multiply-resonant normal form alone would imply perpetual confinement of all chaotic orbits in balls of radius $O\left(\epsilon^{1 / 2}\right)$ in the action space. Nevertheless, this picture is altered due to the effects of the remainder which eventually causes the orbits to diffuse away of their initial $O\left(\epsilon^{1 / 2}\right)$ domain. Now, via a sequence of hamiltonian normalization steps we find that there is an optimal order at which the size of the remainder becomes exponentially small in a power of $1 / \epsilon$. This, in turn, implies an exponentially small semi-analytic upper bound of the value of the diffusion coefficient $D$. Unfortunately, such a bound turns usually to be very unrealistic, as it overestimates by a large factor the true value of $D$ (or, equivalently, it underestimates the time of practical stability). We are thus led to conclude that, whereas the remainder $R$ constitutes a quantity of primary interest in quantitative applications of Nekhoroshev theory, the precise relation between $R$ and $D$ is apparently very different from what upper bound estimates would suggest. Instead, a detailed analysis of the effects of the remainder on dynamics appears to be necessary in order to formulate a more precise theory of the relation between $R$ and $D$.

In the sequel, we present such an analysis in systems of three degrees of freedom. In this analysis, we still have to rely on an assumption for which numerical indications are available, namely that the local character of diffusion in sufficiently small domains of the action space is 'normal', that is, the mean square spread of the actions of the chaotic orbits grows linearly with time (there are indications that global diffusion, which concerns ensembles or orbits diffusing in a substantial part of the Arnold web over much longer timescales, could also be described as 'normal' (see [33]); however, the issue of the laws of global diffusion can only be hoped to tackle after the laws of local diffusion have been adequately understood). In the rest of our analysis, we proceed by expressing all quantities of interest in terms of the remainder function, which, in turn, is calculated in concrete examples by a well-defined algebraic procedure. Finally, we estimate via this analysis how $D$ depends on the size $\left\|R_{o p t}\right\|$ of the remainder at the optimal normalization order. It should be noted that the idea that the stability properties of the orbits in nearly-integrable systems depend on the size of the optimal remainder is not new, but it is one permeating nearly all forms of canonical perturbation theory. The novel feature here, instead, is to use $\left\|R_{\text {opt }}\right\|$ not as an upper bound for $D$, but as a way to estimate $D$ via examining the relation between the two quantities as determined by independent numerical experiments. One main prediction is that this relation is altered according to the multiplicity of resonance conditions holding in the action domain of interest. More concretely, we predict that the diffusion coefficient $D$ scales with $\left\|R_{\text {opt }}\right\|$ 
as a power-law $D \propto\left\|R_{\text {opt }}\right\|^{p}$, where $p \simeq 2$ in doubly-resonant domains, while $p=2(1+b)$ in simply resonant domains, for some constant $b>0$. A combination of theoretical arguments found in [9] 11], together with quantitative estimates on the relation between the size of the socalled separatrix splitting (see subsection 2.3.2) and the normal form remainder given in [49], suggest $b \simeq 0.5$, i.e. $p \simeq 3$ in simply resonant domains. This agrees with the numerical results obtained in a previous study [20].

In [20], a computer-algebraic program was written in order to calculate the optimal normal form as well as the remainder function $R_{o p t}$ at the optimal mormalization order in a case of simple resonance, employing the same Hamiltonian model as in [38]. This operation involved computing about $5 \times 10^{7}$ Fourier coefficients, at a truncation order in Fourier space as high as $K=44$. Comparing the computed size of $\left\|R_{\text {opt }}\right\|$ versus available numerical data on $D$ from [38], the scaling $D \propto\left\|R_{\text {opt }}\right\|^{2.98}$ was found by numerical fitting. In the present paper, after presenting some theoretical results, we make a similar numerical calculation as in [20] but in the case of a double resonance. In order to reach the optimal normalization, we had to extend all normal form calculations up to the Fourier order $K=50\left(8 \times 10^{7}\right.$ coefficients $)$. We thus determined the size of the optimal remainder $\left\|R_{\text {opt }}\right\|$ for many different values of the small parameter $\epsilon$. In the same time, we computed the diffusion coefficient $D$ for the same values of $\epsilon$ by a purely numerical procedure involving runs of ensembles of chaotic orbits (see section 3 ). Finally, we made two independent numerical comparisons of the relation between $D$ and $\left\|R_{o p t}\right\|$. The latter yield the power laws $D \propto\left\|R_{o p t}\right\|^{2.1}$ and $D \propto\left\|R_{o p t}\right\|^{2.3}$ respectively. This essentially confirms that $p$ is close to 2 in doubly resonant domains, albeit with a small noticeable difference even in this case, which probably requires a more precise theory to interpret.

Besides the above computation, our analysis using high order normal forms resulted in a relevant result regarding the possibility to visualize how the phenomenon of Arnold diffusion proceeds locally, within a doubly-resonant domain, by materializing the computation of a convenient set of variables helping to this purpose, that were proposed in the work [4]. We note that numerical evidence for Arnold diffusion of orbits entering from simple to double resonances was presented in [33]. Here, we provide a detailed topological description of this phenomenon. The whole computation consists of: i) computing a set of resonant canonical action-angle variables via a sequence of Lie canonical transformations, ii) taking a 2D Poincaré surface of section of the doubly-resonant normal form dynamics (which represents a system of two degrees of freedom), and (more importantly) iii) using the energy $E_{Z}$ of the normal form as the third variable, showing the effect of Arnold diffusion. According to theory, the value of $E_{Z}$ changes exponentially slowly in time due to the effect of the remainder. In the sequel we refer to this phenomenon as 'drift', although in reality it means that a number of quantities can be characterized as undergoing random walk during the whole diffusion process. Besides setting the timescale of diffusion, the drift can be viewed also as the source of a dynamical phenomenon, namely the communication between chaotic domains that would be otherwise isolated under the doubly-resonant normal form hamiltonian flow. We show in a true example the excursion of a chaotic orbit within the doubly-resonant domain as it appears in the above proposed set of variables. We thus identify a sequence of chaotic transitions of such an orbit from one resonant domain to another. In fact, in each transition the orbit bypasses the barriers imposed by normal form dynamics via a 'third dimension', i.e. the slowly drifting value of $E_{Z}$. We finally argue that, besides their practical utility, such illustrations are also suggestive of the geometric structure underlying the asymptotic manifolds of lower-dimensional tori filling the phase space in the domain of a double resonance. These manifolds are important, because, following the spirit of Arnold's original work [2], it has been widely conjectured that their heteroclinic intersections constitute a primary cause of 
Arnold diffusion. Of course, proving this fact represents a well known important open problem of dynamical systems' theory.

The structure of the paper is as follows: Section 2 presents the theory, focusing on the normal form algorithm, multiply-resonant dynamics, effect of the remainder, and, finally, on the relation between $D$ and $\left\|R_{\text {opt }}\right\|$. We describe in some length all necessary theoretical steps in order to render the paper as self-contained as possible. Section 3 then passes to the numerical results. We present i) the results from the normal form computer-algebraic construction, ii) the visualization of Arnold diffusion using appropriate variables based on the normal form computation, iii) the numerical calculation of the diffusion coefficient $D$, and, finally iv) the comparison of $D$ with $\left\|R_{\text {opt }}\right\|$. Section 4 summarizes the main conclusions of the present study.

\section{Theory}

Most statements made in subsections 2.1 and 2.2 below, regarding the properties of the Hamiltonian models considered as well as the algorithm by which we perform Hamiltonian normalization, are applicable to systems of an arbitrary number of degrees of freedom. In order, however, to be consistent with the rest of the paper, we use everywhere a notation referring to systems of three degrees of freedom. On the other hand, the analysis of subsection 2.3 applies to the study of the diffusion in doubly or simply resonant domains. In systems of three degrees of freedom, the latter represent the only possible multiplicities of a resonance condition, while in systems of more than three degrees of freedom there are also cases of intermediate resonance multiplicities between one and the maximal. The latter's study, nevertheless, is well beyond our present computational capacity, and thus it is left as an open problem.

\subsection{Definitions}

We consider three degrees of freedom systems of the form (1), where $H$ satisfies the following analyticity and convexity conditions:

i) Analyticity: $H$ is assumed to be an analytic function in a complexified domain of its arguments. Namely, we assume that there is an open domain $\mathcal{I} \subset \mathbf{R}^{3}$ and a positive number $\rho$ such that for all points $I_{*} \equiv\left(I_{1 *}, I_{2 *}, I_{3 *}\right) \in \mathcal{I}$ and all complex quantities $I_{i}^{\prime} \equiv I_{i}-I_{i *}$ satisfying the inequalities $\left|I_{i}^{\prime}\right|<\rho$, the function $H_{0}$ admits a convergent Taylor expansion

$$
H_{0}=H_{0 *}+\omega_{*} \cdot I^{\prime}+\sum_{i=1}^{3} \sum_{j=1}^{3} \frac{1}{2} M_{i j *} I_{i}^{\prime} I_{j}^{\prime}+\ldots
$$

where $\omega_{*}=\nabla_{I} H_{0}\left(I_{*}\right)$, and $M_{i j *}$ are the entries of the Hessian matrix of $H_{0}$ at $I_{*}$. Furthermore, we assume that there is a positive constant $\sigma$ such that for all $I \in \mathcal{I}, H_{1}$ admits an absolutely convergent Fourier expansion

$$
H_{1}=\sum_{k} h_{k}(I) \exp (i k \cdot \phi)
$$

in a domain where all three angles satisfy $0 \leq \operatorname{Re}\left(\phi_{i}\right)<2 \pi,\left|\operatorname{Im}\left(\phi_{i}\right)\right| \leq \sigma$. By the Fourier theorem (see e.g. [30]), this condition implies that the coefficients $h_{k}(I)$ decay exponentially with the $L^{1}$-modulus $|k| \equiv\left|k_{1}\right|+\left|k_{2}\right|+\left|k_{3}\right|$, that is, there is a positive constant $A$ such that the bound

$$
\left|h_{k}(I)\right|<A \exp (-|k| \sigma)
$$


holds for all $k \in \mathcal{Z}^{3}$. We finally assume that all coefficients $h_{k}$ admit Taylor expansions with respect to $I_{*}$

$$
h_{k}=h_{k *}+\nabla_{I_{*}} h_{k} \cdot I^{\prime}+\frac{1}{2} \sum_{i=1}^{3} \sum_{j=1}^{3} h_{k, i j *} I_{i}^{\prime} I_{j}^{\prime}+\ldots
$$

(where $h_{k, i j *}$ are the entries of the Hessian matrix of $h_{k}(I)$ at $I_{*}$ ), which are convergent in the same union of domains as for $H_{0}$.

ii) Convexity: For the Hessian matrix $M_{*}$, which is real symmetric, we assume a simple quasiconvexity condition, namely that for all $I_{*} \in \mathcal{I}$ either two of the (real) eigenvalues of $M_{*}$ have the same sign and one is equal to zero, or all three eigenvalues have the same sign. Furthermore, we define two constants:

$$
\mu_{\min }=\min \left\{\left|\mu_{j}\right|\right\}, \quad \mu_{\max }=\max \left\{\left|\mu_{j}\right|\right\}
$$

where $j$ is a label of only non-zero eigenvalues $\mu_{j}$ of $M_{*}$, i.e. $j=1,2$ or $j=1,2,3$ if there are two or three non-zero eigenvalues respectively.

As will be discussed in detail in subsection 2.3 , the quasi-convexity condition is essential, since it introduces a confinement of the orbits for exponentially long times on a surface arising from the condition of preservation of the energy (see [4]).

We now give some definitions allowing to characterize resonant dynamics.

A resonant manifold $\mathcal{R}_{k}$ associated with a non-zero wavevector $k$ with co-prime integer components $k \equiv\left(k_{1}, k_{2}, k_{3}\right)$ is the two-dimensional locus defined by

$$
\mathcal{R}_{k}=\left\{I \in \mathcal{I}: k_{1} \omega_{1}(I)+k_{2} \omega_{2}(I)+k_{3} \omega_{3}(I)=0\right\},
$$

where $\omega_{i}(I)=\partial H_{0} / \partial I_{i}$.

Let $I_{*} \in \mathcal{I}$ be such that all three frequencies $\omega_{i}\left(I_{*}\right), i=1,2,3$ are different from zero. We now distinguish the following three cases:

i) Non-resonance: no resonant manifold $\mathcal{R}_{k}$ contains $I_{*}$.

ii) Simple resonance: one resonant manifold $\mathcal{R}_{k}$ contains $I_{*}$.

iii) Double resonance: more than one resonant manifolds contain $I_{*}$. In the latter case, it is possible to choose two linearly independent vectors $k^{(1)}, k^{(2)}$ such that all resonant manifolds $\mathcal{R}_{k}$ containing $I_{*}$ are labeled by vectors $k$ which are linear combinations of the chosen vectors $k^{(1)}, k^{(2)}$ with rational coefficients. The intersection of these manifolds forms a one-dimensional resonant junction. A doubly-resonant point $I_{*}$ always corresponds to the intersection of a resonant junction with a constant energy surface $H_{0}\left(I_{*}\right)=E$.

In the above definitions, resonant manifolds $\mathcal{R}_{k}$ of all possible wavevectors $k$ have been considered. It is well known, however, that in normal form theory a natural truncation limit $|k|<K$ arises in Fourier space (see below). Accounting for this possibility, we call a point $I_{*} \in \mathcal{I}$ i) non-resonant, ii) simply resonant, or iii) doubly resonant with respect to a $K$-truncation, if the number of resonant manifolds $\mathcal{R}_{k}$ with $|k|<K$ passing through $I_{*}$ are i) zero, ii) one and iii) more than one respectively.

Finally, it will be convenient to introduce a definition concerning open domains in $\mathcal{I}$. Let $W_{I_{*}, B}$ be a ball of radius $B$ around one point $I_{*}$ in $\mathcal{I}$. If $H_{0}$ satisfies convexity conditions as assumed above, for $B$ small whatsoever the domain $\mathcal{W}_{I_{*}, B}$ is crossed by a dense set of resonant manifolds $\mathcal{R}_{k}$. However, for any fixed value of the positive integer $K$, only a finite subset of 
the manifolds $\mathcal{R}_{k}$ satisfy $|k|<K$. The domain $\mathcal{W}_{I_{*}, B}$ is then called: i) non-resonant, ii) simplyresonant, and iii) doubly-resonant with respect to the $K$-truncation if $I_{*}$ is, respectively, nonresonant, simply-resonant or doubly-resonant, and no other resonant manifolds $\mathcal{R}_{k}$ with $|k|<K$ cross $\mathcal{W}_{I_{*}, B}$ except for the ones passing through $I_{*}$.

\subsection{Normal form construction}

All our estimates on the speed of diffusion are based on an appropriate normal form construction. In this, we adopt the method exposed in detail in [20], which lends itself conveniently to i) developing a computer-algebraic program, and ii) deriving analytical estimates on the size of various quantities appearing in the course of Hamiltonian normalization. The main elements of this method are:

Expansion centers. The action space can be covered by domains $\mathcal{W}_{I_{*}, B}$, centered around points $I_{*}$ which serve as expansion centers of both the original Hamiltonian and the normal form. We choose the points $I_{*}$ to belong to the set of all doubly-resonant points of $\mathcal{I}$, denoted by $\mathcal{D}$, and by setting $B$ as of order $O\left(\epsilon^{1 / 2}\right)$. The covering is possible because $\mathcal{D}$ is dense in $\mathcal{I}$. A normal form construction as done below is valid within one domain $\mathcal{W}_{I_{*}, B}$ (this is essentially the same starting point as in Lochak's [45] analytic construction leading to a proof of the Nekhoroshev theorem). A crucial remark is that the characterization of dynamics within $W_{I_{*}, B}$ as non resonant, simply resonant, or doubly resonant depends on $\epsilon$. This is because, as shown below, the optimal normal form truncation order $K=K_{\text {opt }}$ in Fourier space depends on the value of $\epsilon$. Furthermore, for a given value of $K$, the set $\mathcal{D}$ can be decomposed in three disjoint sets $\mathcal{D}=\mathcal{D}_{0, K} \cup \mathcal{D}_{1, K} \cup \mathcal{D}_{2, K}$, containing all non-resonant, simply resonant and doubly resonant points respectively with respect to the $K$-truncation. Thus, the characterization of resonant dynamics within $\mathcal{W}_{I_{*}, B}$ depends on whether, according to the value of $K, I_{*}$ belongs to $\mathcal{D}_{0, K}, \mathcal{D}_{1, K}$, or $\mathcal{D}_{2, K}$.

Resonant module: Let $I_{*}$ be a point of $\mathcal{D}$ and $k^{(1)} \equiv\left(k_{1}^{(1)}, k_{2}^{(1)}, k_{3}^{(1)}\right), k^{(2)} \equiv\left(k_{1}^{(2)}, k_{2}^{(2)}, k_{3}^{(2)}\right)$ two linearly independent vectors such that $k^{(i)} \cdot \omega\left(I_{*}\right)=0$ for $i=1,2$. More than one choices of $k^{(1)}$ and $k^{(2)}$ are possible. In the sequel we choose $k^{(1)}$ and $k^{(2)}$ so that $\left|k^{(1)}\right|+\left|k^{(2)}\right|$ is minimal. The vector $m \equiv\left(m_{1}, m_{2}, m_{3}\right)$ defined by

$$
m_{1}=k_{2}^{(1)} k_{3}^{(2)}-k_{2}^{(2)} k_{3}^{(1)}, \quad m_{2}=k_{3}^{(1)} k_{1}^{(2)}-k_{3}^{(2)} k_{1}^{(1)}, \quad m_{3}=k_{1}^{(1)} k_{2}^{(2)}-k_{1}^{(2)} k_{2}^{(1)}
$$

is parallel to the vector $\omega\left(I_{*}\right)$ since $k \cdot m=0$ for all $k$ satisfying $k \cdot \omega\left(I_{*}\right)=0$. If $m_{1}, m_{2}, m_{3}$ are not co-prime integers, we re-define $m$ by dividing the $m_{i}$ by their greatest common divisor. The set

$$
\mathcal{M} \equiv\left\{k \in \mathcal{Z}^{3}: k \cdot m=0\right\}
$$

is hereafter called the resonant module associated with the point $I_{*} \in \mathcal{D}$. The resonant module includes wavevectors $k$ whose respective trigonometric terms $\exp (i k \cdot \phi)$ are to be retained in the normal form.

Action re-scaling: From now on we focus on the construction of the normal form in one specific domain $\mathcal{W}_{I_{*}, B}$. It has been mentioned already that it is convenient to choose $B$ as a quantity scaling proportionally to $\epsilon^{1 / 2}$. The simplest way to accommodate such a choice is by introducing the following re-scaling of all action variables within $\mathcal{W}_{I_{*}, B}$ :

$$
J_{i}=\epsilon^{-1 / 2}\left(I_{i}-I_{i *}\right)=\epsilon^{-1 / 2} I_{i}^{\prime}, \quad i=1,2,3 .
$$


This re-scaling greatly simplifies the normal form algorithm, because it formally removes all terms besides linear in the actions from the kernel of the so-called homological equation (see below, or [20] for details) by which the normalizing generating functions are determined. Eq. (10) does not define a canonical transformation. However, the correct equations of motion in the variables $(J, \phi)$ are produced by the Hamiltonian function $h(J, \phi)=\epsilon^{-1 / 2} H\left(I_{*}+\epsilon^{1 / 2} J, \phi\right)$, i.e. (neglecting a constant)

$$
\begin{aligned}
h(J, \phi) & =\omega_{*} \cdot J+\epsilon^{1 / 2} \sum_{i=1}^{3} \sum_{j=1}^{3} \frac{1}{2} M_{i j *} J_{i} J_{j}+\ldots \\
& +\epsilon^{1 / 2} \sum_{k}\left(h_{k *}+\epsilon^{1 / 2} \nabla_{I_{*}} h_{k} \cdot J+\frac{\epsilon}{2} \sum_{i=1}^{3} \sum_{j=1}^{3} h_{k, i j *} J_{i} J_{j}+\ldots\right) \exp (i k \cdot \phi)
\end{aligned}
$$

where the first line in the above equation comes from the integrable part $H_{0}$ of the original Hamiltonian (Eq.(2)), while the second line comes from the perturbation $H_{1}$ (Eq.(3)) given the series expansion of the Fourier coefficients as in Eq. (5).

Book-keeping: We now split the Hamiltonian (11) in parts of different order of smallness, which are to be normalized step by step. The function (11) contains terms of various orders in the small parameter $\epsilon^{1 / 2}$. However, the presence of a second 'small parameter' $e^{-\sigma}$ is implied in (11) by the exponential decay of all Fourier coefficients $h_{k *}, h_{k, i j *}$, etc., due to Eq. (4) (see [30] pp.90-91 for a thorough exposition of the role of this small parameter in Nekhoroshev theory). We take both parameters into account by introducing an integer $K^{\prime}$ such that $e^{-\sigma K^{\prime}} \sim \epsilon^{1 / 2}$, i.e. by setting:

$$
K^{\prime}=\left[-\frac{1}{2 \sigma} \ln (\epsilon)\right] \text {. }
$$

Using $K^{\prime}$, the Hamiltonian (11) can be split in groups of practically the same order of smallness. This is realized by artificially introducing a 'book-keeping' coefficient $\lambda^{p}$ in front of each term in (11), whose numerical value is set equal to unity at the end of the calculation. Furthermore, for a term of the form $\epsilon^{\mu / 2} f(J) \exp (i k \cdot \phi)$ we set $p=\left[|k| / K^{\prime}\right]+\mu$.

Regarding the above 'book-keeping' process it is worth noting the following: i) This way of splitting the Hamiltonian in different orders of smallness results in a finite number of terms appearing in every power of $\lambda$. ii) This technique is suggested already by Poincaré [53] and Arnold [1]. In fact, the dependence of $K^{\prime}$ on $\epsilon$ is weak, since it is logarithmic, so that an alternative choice to the 'ansatz' (12) is to set $K^{\prime}=$ const $\sim 1 / \sigma$. In fact, according to Giorgilli [30] this is an optimal choice. iii) Since, at every normalization order, we have a reduction of the analyticity domain, one could consider re-defining $K^{\prime}$ at every normalization step. However, this is hardly tractable from an algorithmic point of view. Instead, keeping $K^{\prime}$ constant at all normalization orders should be viewed as a rule indicating the sequence by which the various terms in the Hamiltonian are normalized, i.e., the terms or order $\lambda^{r}$ are normalized in the r-th step. Albeit not necessarily optimal regarding the grouping of the terms according to their size, this rule proves simple to implement and sufficient in practice.

Returning to the form of the Hamiltonian after introducing the book-keeping factor $\lambda$, the Hamiltonian reads:

$$
h=\omega_{*} \cdot J+\lambda \epsilon^{1 / 2} \sum_{i=1}^{3} \sum_{j=1}^{3} \frac{1}{2} M_{i j *} J_{i} J_{j}+\ldots+\sum_{k}\left(\lambda^{1+\left[|k| / K^{\prime}\right]} \epsilon^{1 / 2} h_{k *}\right.
$$




$$
\left.+\lambda^{2+\left[|k| / K^{\prime}\right]} \epsilon \nabla_{I_{*}} h_{k} \cdot J+\lambda^{3+\left[|k| / K^{\prime}\right]} \frac{\epsilon^{3 / 2}}{2} \sum_{i=1}^{3} \sum_{j=1}^{3} h_{k, i j *} J_{i} J_{j}+\ldots\right) \exp (i k \cdot \phi)
$$

Setting $Z_{0}=\omega_{*} \cdot J$, the Hamiltonian (13) resumes the form

$$
h=H^{(0)}(J, \phi)=Z_{0}+\sum_{s=1}^{\infty} \lambda^{s} H_{s}^{(0)}\left(J, \phi ; \epsilon^{1 / 2}\right)
$$

where i) the superscript ( 0 ) denotes zeroth-step of the normalization procedure (= original Hamiltonian), ii) the exponent of $\lambda$ in different terms keeps track of their true order of smallness, and iii) the functions $H_{s}^{(0)}$ are of the form

$$
H_{s}^{(0)}=\sum_{\mu=1}^{s} \epsilon^{\mu / 2} \sum_{|k|=K^{\prime}(s-\mu)}^{K^{\prime}(s-\mu+1)-1} H_{\mu, k}^{(0)}(J) \exp (i k \cdot \phi)
$$

where $H_{\mu, k}^{(0)}(J)$ are polynomials containing terms of degree $\mu-1$ or $\mu$ in the action variables $J$. Precisely, we have:

$$
H_{\mu, k}^{(0)}(J)=\sum_{\mu_{1}=0}^{\mu-1} \sum_{\mu_{2}=0}^{\mu-1-\mu_{1}} \sum_{\mu_{3}=0}^{\mu-1-\mu_{1}-\mu_{2}} \frac{1}{\mu_{1} ! \mu_{2} ! \mu_{3} !} \frac{\partial^{\mu-1} h_{k}\left(I_{*}\right)}{\partial^{\mu_{1}} I_{1} \partial^{\mu_{2}} I_{2} \partial^{\mu_{3}} I_{3}} J_{1}^{\mu_{1}} J_{2}^{\mu_{2}} J_{3}^{\mu_{3}}
$$

if $|k|>0$, or

$$
\begin{aligned}
& H_{\mu, k}^{(0)}(J)=\sum_{\mu_{1}=0}^{\mu} \sum_{\mu_{2}=0}^{\mu-\mu_{1}} \sum_{\mu_{3}=0}^{\mu-\mu_{1}-\mu_{2}} \frac{1}{\mu_{1} ! \mu_{2} ! \mu_{3} !} \frac{\partial^{\mu} H_{0}\left(I_{*}\right)}{\partial^{\mu_{1}} I_{1} \partial^{\mu_{2}} I_{2} \partial^{\mu_{3}} I_{3}} J_{1}^{\mu_{1}} J_{2}^{\mu_{2}} J_{3}^{\mu_{3}} \\
& +\sum_{\mu_{1}=0}^{\mu-1} \sum_{\mu_{2}=0}^{\mu-1-\mu_{1}} \sum_{\mu_{3}=0}^{\mu-1-\mu_{1}-\mu_{2}} \frac{1}{\mu_{1} ! \mu_{2} ! \mu_{3} !} \frac{\partial^{\mu-1} h_{0}\left(I_{*}\right)}{\partial^{\mu_{1}} I_{1} \partial^{\mu_{2}} I_{2} \partial^{\mu_{3}} I_{3}} J_{1}^{\mu_{1}} J_{2}^{\mu_{2}} J_{3}^{\mu_{3}}
\end{aligned}
$$

if $k=0$.

Hamiltonian normalization: We use the algorithm of composition of Lie series in order to perform the Hamiltonian normalization. Let us recall that the purpose of the normalization is to introduce a sequence of canonical transformations $(J, \phi) \equiv\left(J^{(0)}, \phi^{(0)}\right) \rightarrow\left(J^{(1)}, \phi^{(1)}\right)$ $\rightarrow\left(J^{(2)}, \phi^{(2)}\right) \rightarrow \ldots$ so that the Hamiltonian expressed as a function of the new variables allows one to more easily identify the main features of dynamics. After $r$ normalization steps, the old variables $(J, \phi) \equiv\left(J^{(0)}, \phi^{(0)}\right)$ are expressed in terms of the new variables $\left(J^{(r)}, \phi^{(r)}\right)$, and the Hamiltonian $H^{(r)}\left(J^{(r)}, \phi^{(r)}\right)=h\left(J\left(J^{(r)}, \phi^{(r)}\right), \phi\left(J^{(r)}, \phi^{(r)}\right)\right)$ takes the form

$$
H^{(r)}\left(J^{(r)}, \phi^{(r)}\right)=Z^{(r)}\left(J^{(r)}, \phi^{(r)} ; \lambda, \epsilon\right)+R^{(r)}\left(J^{(r)}, \phi^{(r)} ; \lambda, \epsilon\right)
$$

The terms $Z^{(r)}\left(J^{(r)}, \phi^{(r)} ; \lambda, \epsilon\right)$ and $R^{(r)}\left(J^{(r)}, \phi^{(r)} ; \lambda, \epsilon\right)$ are called the normal form and the remainder respectively. The normal form is a finite expression which contains terms up to order $r$ in the book-keeping constant $\lambda$, while the remainder is a series containing terms of order $\lambda^{r+1}$ and beyond. The mathematical structure of the normal form term $Z^{(r)}$ is such as to imply an easily identifiable dynamics in the variables $\left(J^{(r)}, \phi^{(r)}\right)$ (e.g. an oscillator or pendulum dynamics). On 
the other hand, the remainder is a convergent series in a restriction of the domain of analyticity of the original Hamiltonian, which represents a perturbation with respect to the Hamiltonian flow of $Z^{(r)}$. An optimal normalization order $r_{\text {opt }}$ exists (see below) where the process must be stopped.

The Hamiltonian normalization is implemented step-by-step by the recursive equation:

$$
H^{(r)}=\exp \left(L_{\chi_{r}}\right) H^{(r-1)}
$$

where $\chi_{r}$ is the r-th step Lie generating function and $L_{\chi_{r}} \equiv\left\{\cdot, \chi_{r}\right\}$ is the Poisson bracket operator. Both $H^{(r)}$ and $\chi_{r}$ are functions of the variables $J^{(r)}, \phi^{(r)}$. The generating function is defined by the solution of the homological equation

$$
\left\{\omega_{*} \cdot J^{(r)}, \chi_{r}\right\}+\tilde{H}_{r}^{(r-1)}\left(J^{(r)}, \phi^{(r)}\right)=0
$$

where $\tilde{H}_{r}^{(r-1)}\left(J^{(r)}, \phi^{(r)}\right)$ denotes all terms of $H^{(r-1)}$ which i) have a book-keeping coefficient $\lambda^{r}$ in front, and ii) belong to the range of the operator $\left\{\omega_{*} \cdot J^{(r)}, \cdot\right\}$. Given the definition of the resonant module $\mathcal{M}$ in Eq.(9), one has the relation

$$
\tilde{H}_{r}^{(r-1)}=H_{r}^{(r-1)}-Z_{r}
$$

where $H_{r}^{(r-1)}$ are all the terms of $H^{(r-1)}$ having a factor $\lambda^{r}$, and $Z_{r}$ are the normal form terms of $H_{r}^{(r-1)}$, that is all the trigonometric terms whose wavevectors $k$ belong to $\mathcal{M}$. It follows immediately that $H^{(r)}$ has the form

$$
H^{(r)}=Z_{0}+Z_{1}+\ldots+Z_{r}+R^{(r)}
$$

where all terms in the functions $Z_{i}$ have a factor $\lambda^{i}$, while $R^{(r)}$ is a series in powers of $\lambda$ starting with terms of order $\lambda^{r+1}$.

Optimal truncation: In the analytical part of the Nekhoroshev theory it is demonstrated that the whole normalization process has an asymptotic character. Namely, i) the domain of convergence of the remainder series $R^{(r)}$ shrinks as the normalization order $r$ increases, and ii) the size $\left\|R^{(r)}\right\|$ of $R^{(r)}$, where $\|\cdot\|$ is a properly defined norm in the space of trigonometric polynomials (see below), initially decreases, as $r$ increases, up to an optimal order $r_{\text {opt }}$ beyond which $\left\|R^{(r)}\right\|$ increases with $r$. In the Nekhoroshev regime, one has $\left\|Z^{\left(r_{o p t}\right)}\right\|>>\left\|R^{\left(r_{o p t}\right)}\right\|$. Thus, stopping at $r_{o p t}$ best unravels the dynamics, which is given essentially by the Hamiltonian flow of $Z^{\left(r_{o p t}\right)}$ slightly perturbed by $R^{\left(r_{o p t}\right)}$. The long term consequences of this perturbation, which determine the speed of diffusion, will be analyzed in subsection 2.3.

The normal form $Z^{(r)}=Z_{0}+Z_{1}+\ldots Z_{r}$ contains trigonometric terms $\exp (i k \cdot \phi)$ of order not greater than $K=K^{\prime} r-1$. Let $r_{\text {opt }}$ be the optimal normalization order. It is well known that the dependence of $r_{\text {opt }}$ on $\epsilon$ is given by an inverse power-law, namely

$$
r_{\text {opt }} \sim \epsilon^{-a}
$$

The exponents $1 / 6,1 / 4$ and $1 / 2$, referring to the non-resonant, simply resonant, and doubly resonant normal form constructions respectively, are found in [54]. We emphasize that, while, due to the introduction of the book-keeping process, the algorithm of Hamiltonian normalization analyzed above is not technically identical with the usual normalization procedure used in the proof of the Nekhoroshev theorem (e.g. as in [54]), in practice we recover the estimate (21), and the resulting exponents, both in the simply resonant case (see [20]) and in the doubly resonant case, as confirmed by numerical experiments in section 3 below. In particular, we find that since 
the leading terms in the remainder are $O\left(\lambda^{r_{\text {opt }}+1}\right)$, the size of the remainder is of order $O\left(\epsilon^{\left(r_{o p t}+1\right) / 2}\right)$, implying (viz.Eq.(12)):

$$
\left\|R^{\left(r_{o p t}\right)}\right\| \sim \epsilon^{1 / 2} \exp \left(\frac{-K^{\prime} \sigma}{\epsilon^{a}}\right)
$$

i.e. the remainder is exponentially small in $1 / \epsilon$ in accordance with the Nekhoroshev theorem. The Fourier order

$$
K_{\text {opt }}(\epsilon)=K^{\prime} r_{o p t}(\epsilon)
$$

is hereafter called the optimal $\mathrm{K}$-truncation order. All the normal form terms of $H^{\left(r_{o p t}\right)}$ have Fourier orders satisfying $|k|<K_{\text {opt }}(\epsilon)$.

\subsection{Resonant normal form dynamics and the rate of diffusion}

We are now in a position to discuss the essence of all the previous definitions. The key point is to observe that, depending on the value of $\epsilon$, the same expansion point $I_{*} \in \mathcal{D}$ of the normal form construction turns to be either non-resonant, or simply or doubly resonant with respect to the optimal K-truncation. In particular, let $k^{(1)}$ and $k^{(2)}$ be two linearly independent vectors of $\mathcal{M}$ such that for all $k \in \mathcal{M}$ one has $|k| \geq\left|k^{(2)}\right| \geq\left|k^{(1)}\right|$. We then distinguish the following three regimes: i) $\left|k^{(2)}\right|<K_{\text {opt }}(\epsilon)$. Then, the point $I_{*}$ is doubly-resonant with respect to the optimal K-truncation. This is the case we mainly focus on in the sequel. The main theoretical results are given in subsection 2.3.1, while the main numerical results are given in section 3. ii) $\left|k^{(1)}\right|<K_{o p t}(\epsilon) \leq\left|k^{(2)}\right|$. Then, $I_{*}$ is simply-resonant with respect to the optimal K-truncation. One such example was dealt with in the numerical study [20]. Further theoretical analysis of this case is made in subsection 2.3.2. iii) $K_{o p t}(\epsilon)<\left|k^{(1)}\right| \leq\left|k^{(2)}\right|$. Then, $I_{*}$ is non-resonant with respect to the optimal K-truncation. Since $K_{o p t}$ decreases as $\epsilon$ increases, for fixed $\left|k^{(1)}\right|+\left|k^{(2)}\right|$ this inequality always occurs if $\epsilon>\epsilon_{1}$, where $\epsilon_{1}$ is a threshold depending on $k^{(1)}, k^{(2)}$. The case $\epsilon_{1}>\epsilon_{c}$, where $\epsilon_{c}$ is the critical threshold for the onset of the Nekhoroshev regime, presents no practical interest. If, however, $\epsilon_{1}<\epsilon_{c}$, then, for all values of $\epsilon$ in the interval $\epsilon_{1}<\epsilon<\epsilon_{c}$ the optimal normal form describes a true non-resonant dynamics. Note that in order to describe the dynamics close to a point $I_{*}^{\prime}$ of the action space corresponding to Diophantine frequencies $\omega_{*}^{\prime}$, it suffices to choose $I_{*}$ such that $\omega_{*}$ corresponds to a very high order rational approximation of $\omega_{*}$, i.e. the numbers $\left(\omega_{1 *}, \omega_{2 *}, \omega_{3 *}\right)$ are high order finite digit approximants of the numbers $\left(\omega_{1 *}^{\prime}, \omega_{2 *}^{\prime}, \omega_{3 *}^{\prime}\right)$. Then, $\left|k^{(1)}\right|+\left|k^{(2)}\right|$ becomes very large, and $\epsilon_{1}$ approaches very close to zero. In this case, for $\epsilon$ sufficiently small, we expect the existence of a set of points of large measure within $\mathcal{W}_{I_{x}, B}$, corresponding to Kolmogorov - Arnold - Moser tori in the neighborhood of the point $I_{*}$. However, these tori cannot fill an open domain. Thus, the diffusion in action space is topologically possible for (very weakly) chaotic orbits wandering through the set of KAM tori. However, in the absence of significant resonant chaotic layers (since no important resonances cross $\mathcal{W}_{I_{*}, B}$ ), the question of whether or not the diffusion can be observed is of no practical interest, since its rate would be extremely slow to be of any relevance in applications. Thus, the non-resonant case is no further considered below.

\subsubsection{Double resonance}

As long as $\left|k^{(2)}\right|<K_{\text {opt }}(\epsilon)$, the point $I_{*}$ is doubly-resonant with respect to the optimal Ktruncation. In this case, the normal form contains either terms independent of the angles, or trigonometric terms of the form $\exp \left(i k^{(1)} \cdot \phi^{\left(r_{o p t}\right)}\right), \exp \left(i k^{(2)} \cdot \phi^{\left(r_{o p t}\right)}\right)$ and their multiples in the 
exponents. Writing explicitly only the most important terms, the normalized Hamiltonian takes the form:

$$
\begin{aligned}
h\left(J^{\left(r_{o p t}\right)}, \phi^{\left(r_{o p t}\right)}\right) & =Z\left(J^{\left(r_{o p t}\right)}, \phi^{\left(r_{o p t}\right)}\right)+R\left(J^{\left(r_{o p t}\right)}, \phi^{\left(r_{o p t}\right)}\right) \\
& =\omega_{*} \cdot J^{\left(r_{o p t}\right)}+\epsilon^{1 / 2} \sum_{i=1}^{3} \sum_{j=1}^{3} \frac{1}{2} M_{i j *} J_{i}^{\left(r_{o p t}\right)} J_{j}^{\left(r_{o p t}\right)}+\ldots \\
& +\epsilon^{1 / 2} \sum_{n_{1}, n_{2} \in Z^{2}} g_{n_{1}, n_{2}}\left(J^{\left(r_{o p t}\right)}\right) \exp \left(i\left(n_{1} k^{(1)}+n_{2} k^{(2)}\right) \cdot \phi^{\left(r_{o p t}\right)}\right)+\ldots \\
& \left.+R\left(J^{\left(r_{o p t}\right)}, \phi^{\left(r_{o p t}\right)}\right)\right)
\end{aligned}
$$

The main feature of the Hamiltonian (24) is that, since in $\left.Z\left(J^{\left(r_{o p t}\right)}, \phi^{\left(r_{\text {opt }}\right)}\right)\right)$ there are coupling terms between more than one resonant angles, the normal form $Z$ alone is non-integrable. In fact, $Z$ can be decomposed into an integrable system of one degree of freedom and a non-integrable system of two degrees of freedom (see [4]). The decomposition is done by the linear canonical transformation $\left(J_{1}^{\left(r_{o p t}\right)}, J_{2}^{\left(r_{o p t}\right)}, J_{3}^{\left(r_{o p t}\right)}, \phi_{1}^{\left(r_{o p t}\right)}, \phi_{2}^{\left(r_{o p t}\right)}, \phi_{3}^{\left(r_{o p t}\right)}\right) \rightarrow\left(J_{R_{1}}, J_{R_{2}}, J_{F}, \phi_{R_{1}}, \phi_{R_{2}}, \phi_{F}\right)$ defined by

$$
\begin{array}{llll}
J_{1}^{\left(r_{o p t}\right)}=k_{1}^{(1)} J_{R_{1}}+k_{1}^{(2)} J_{R_{2}}+m_{1} J_{F}, & \phi_{R_{1}} & =k_{1}^{(1)} \phi_{1}^{\left(r_{o p t}\right)}+k_{2}^{(1)} \phi_{2}^{\left(r_{o p t}\right)}+k_{3}^{(1)} \phi_{3}^{\left(r_{o p t}\right)} \\
J_{2}^{\left(r_{o p t}\right)}=k_{2}^{(1)} J_{R_{1}}+k_{2}^{(2)} J_{R_{2}}+m_{2} J_{F}, & \phi_{R_{2}}=k_{1}^{(2)} \phi_{1}^{\left(r_{o p t}\right)}+k_{2}^{(2)} \phi_{2}^{\left(r_{o p t}\right)}+k_{3}^{(2)} \phi_{3}^{\left(r_{o p t}\right)} \\
J_{3}^{\left(r_{o p t}\right)}=k_{3}^{(1)} J_{R_{1}}+k_{3}^{(2)} J_{R_{2}}+m_{3} J_{F}, & \phi_{F}=m_{1} \phi_{1}^{\left(r_{o p t}\right)}+m_{2} \phi_{2}^{\left(r_{o p t}\right)}+m_{3} \phi_{3}^{\left(r_{o p t}\right)}
\end{array}
$$

where $m \equiv\left(m_{1}, m_{2}, m_{3}\right)$ has been defined in Eq. (8). The Hamiltonian in the new variables reads (apart from a constant)

$$
h=Z\left(J_{R_{1}}, J_{R_{2}}, J_{F}, \phi_{R_{1}}, \phi_{R_{2}}\right)+R_{o p t}\left(J_{R_{1}}, J_{R_{2}}, J_{F}, \phi_{R_{1}}, \phi_{R_{2}}, \phi_{F}\right)
$$

where

$$
\begin{aligned}
& Z\left(J_{R_{1}}, J_{R_{2}}, J_{F}, \phi_{R_{1}}, \phi_{R_{2}}\right)=\left(\omega_{*} \cdot m\right) J_{F} \\
& +\epsilon^{1 / 2} \sum_{i, j=1}^{3} \frac{1}{2} M_{i j *}\left(k_{i}^{(1)} J_{R_{1}}+k_{i}^{(2)} J_{R_{2}}+m_{i} J_{F}\right)\left(k_{j}^{(1)} J_{R_{1}}+k_{j}^{(2)} J_{R_{2}}+m_{j} J_{F}\right)+\ldots \\
& +\epsilon^{1 / 2} \sum_{n_{1}, n_{2} \in Z^{2}} g_{n_{1}, n_{2}}\left(J_{R_{1}}, J_{R_{2}}, J_{F}\right) \exp \left(i\left(n_{1} \phi_{R_{1}}+n_{2} \phi_{R_{2}}\right)\right)+\ldots
\end{aligned}
$$

and the remainder $R_{\text {opt }}\left(J_{R_{1}}, J_{R_{2}}, J_{F}, \phi_{R_{1}}, \phi_{R_{2}}, \phi_{F}\right)$ is exponentially small in $1 / \epsilon$. Since $\phi_{F}$ is ignorable in $Z, J_{F}$ is an integral under the flow of the normal form. On the other hand, the remaining degrees of freedom $\left(J_{R_{1}}, \phi_{R_{1}}\right)$ and $\left(J_{R_{2}}, \phi_{R_{2}}\right)$ are coupled under the flow of $Z$ due to the trigonometric terms $\exp \left(i\left(n_{1} \phi_{R_{1}}+n_{2} \phi_{R_{2}}\right)\right)$. The main characteristics of motion can be understood by the following remarks 1 :

\footnotetext{
${ }^{1}$ Since many different action symbols appear in the previous and in the subsequent analysis, it helps recalling that throughout the paper all action variables defined by a symbol starting with the letter $I$ refer to non-scaled values, i.e. before the re-scaling of Eq. 10 is implemented, while all action variables defined by a symbol starting with the letter $J$ have re-scaled values, according to Eq.(10). Thus, in the domains considered below, all quantities of the form $I-I_{*}$, where $I_{*}$ is the selected central doubly-resonant point of interest, scale proportionally to $\epsilon^{1 / 2}$, while all actions denoted by a letter $J$ exhibit no scaling with $\epsilon$. Furthermore, all Hamiltonian-type functions denoted by $h, H^{(r)}, Z$, or $R$, are expressed in re-scaled variables; only the original Hamiltonian (Eq.(1) is expressed in non-scaled action variables $I$. Finally, the quantities $E^{\prime}$ (Eq. 307) and $E_{Z}$ (Eq. (67)) scale proportionally to $\epsilon^{1 / 2}$.
} 
i) The constant-valued action $J_{F}$ can be viewed as a parameter in the two degrees of freedom Hamiltonian $Z$. Furthermore, except for the case of some very low resonances satisfying $\left|k^{(1)}\right|<$ $K^{\prime}$, all coefficients $g_{n_{1}, n_{2}}$ in (27) are of order $\epsilon^{1 / 2}$ or higher. Thus, the terms

$$
\begin{aligned}
& Z_{0}\left(J_{R_{1}}, J_{R_{2}} ; J_{F}\right)=\left(\omega_{*} \cdot m\right) J_{F} \\
& +\epsilon^{1 / 2} \sum_{i, j=1}^{3} \frac{1}{2} M_{i j *}\left(k_{i}^{(1)} J_{R_{1}}+k_{i}^{(2)} J_{R_{2}}+m_{i} J_{F}\right)\left(k_{j}^{(1)} J_{R_{1}}+k_{j}^{(2)} J_{R_{2}}+m_{j} J_{F}\right)+\ldots
\end{aligned}
$$

define an 'integrable part' of $Z$, while the remaining terms depending on the resonant angles can be considered as a perturbation.

The terms quadratic in $J_{R_{1}}, J_{R_{2}}$ in the r.h.s. of (28) define the quadratic form

$$
\zeta_{0,2}=\frac{1}{2} \sum_{i, j=1}^{3} M_{i j *}\left(k_{i}^{(1)} J_{R_{1}}+k_{i}^{(2)} J_{R_{2}}\right)\left(k_{j}^{(1)} J_{R_{1}}+k_{j}^{(2)} J_{R_{2}}\right)
$$

In Appendix $\mathrm{A}$ it is demonstrated that, due to the quasi-convexity condition assumed for the Hessian matrix $M_{i j *}$, the quadratic form (29) is positive definite. Thus, the constant level curves of the quantity

$$
E^{\prime}=\left(Z_{0}-\left(\omega_{*} \cdot m\right) J_{F}\right)
$$

on the plane $\left(J_{R_{1}}, J_{R_{2}}\right)$, given by

$$
E^{\prime}=\epsilon^{1 / 2} \sum_{i, j=1}^{3} \frac{1}{2} M_{i j *}\left(k_{i}^{(1)} J_{R_{1}}+k_{i}^{(2)} J_{R_{2}}+m_{i} J_{F}\right)\left(k_{j}^{(1)} J_{R_{1}}+k_{j}^{(2)} J_{R_{2}}+m_{j} J_{F}\right),
$$

are ellipses centered at

$$
\begin{aligned}
& J_{R_{1}, 0}=\frac{\left(k^{(1)} \cdot M_{*} k^{(2)}\right)\left(m \cdot M_{*} k^{(2)}\right)-\left(k^{(2)} \cdot M_{*} k^{(2)}\right)\left(m \cdot M_{*} k^{(1)}\right)}{\left(k^{(1)} \cdot M_{*} k^{(1)}\right)\left(k^{(2)} \cdot M_{*} k^{(2)}\right)-\left(k^{(1)} \cdot M_{*} k^{(2)}\right)^{2}} J_{F} \\
& J_{R_{2}, 0}=\frac{\left(k^{(1)} \cdot M_{*} k^{(2)}\right)\left(m \cdot M_{*} k^{(1)}\right)-\left(k^{(1)} \cdot M_{*} k^{(1)}\right)\left(m \cdot M_{*} k^{(2)}\right)}{\left(k^{(1)} \cdot M_{*} k^{(1)}\right)\left(k^{(2)} \cdot M_{*} k^{(2)}\right)-\left(k^{(1)} \cdot M_{*} k^{(2)}\right)^{2}} J_{F} .
\end{aligned}
$$

(the role of the elliptic structures formed around double resonances in the Nekhoroshev theorem is discussed extensively in [6]). If the higher order terms in the action variables of the development of Eq.(28) are taken into account, the constant energy condition of Eq.(30) yields deformed ellipses on the plane $\left(J_{R_{1}}, J_{R_{2}}\right)$. If $J_{R_{1}} \neq J_{R_{1,0}}$ or $J_{R_{2}} \neq J_{R_{2,0}}$, the slow frequencies $\dot{\phi}_{R_{1}} \equiv \omega_{R_{1}}$, $\dot{\phi}_{R_{2}} \equiv \omega_{R_{2}}$ are non-zero, and they are given by

$$
\begin{aligned}
& \omega_{R_{1}}=\left(k^{(1)} \cdot M_{*} k^{(1)}\right)\left(J_{R_{1}}-J_{R_{1,0}}\right)+\left(k^{(1)} \cdot M_{*} k^{(2)}\right)\left(J_{R_{2}}-J_{R_{2,0}}\right)+\ldots \\
& \omega_{R_{2}}=\left(k^{(1)} \cdot M_{*} k^{(2)}\right)\left(J_{R_{1}}-J_{R_{1,0}}\right)+\left(k^{(2)} \cdot M_{*} k^{(2)}\right)\left(J_{R_{2}}-J_{R_{2,0}}\right)+\ldots
\end{aligned}
$$

On the other hand, due to the definition (25) one has

$$
\omega_{R_{1}}=k^{(1)} \cdot \omega\left(J^{\left(r_{o p t}\right)}\right), \quad \omega_{R_{2}}=k^{(2)} \cdot \omega\left(J^{\left(r_{o p t}\right)}\right), \quad \omega_{F}=m \cdot \omega\left(J^{\left(r_{o p t}\right)}\right)
$$


which is valid for any value of $\left(J_{R_{1}}, J_{R_{2}}, J_{F}\right)$ in the domain of convergence of the series (28). It follows that all the resonant manifolds defined by relations of the form $\left(n_{1} k^{(1)}+n_{2} k^{(2)}\right) \cdot \omega\left(J^{\left(r_{\text {opt }}\right)}\right)=$ 0 intersect any of the planes $\left(J_{R_{1}}, J_{R_{2}}\right)$ corresponding to a fixed value of $J_{F}$. Using the notation

$$
\Delta J_{R_{i}}=J_{R_{i}}-J_{R_{i, 0}}, \quad a_{i j}=k^{(i)} \cdot M_{*} k^{(j)}, \quad i, j=1,2
$$

the intersection of one resonant manifold with the plane $\left(J_{R_{1}}, J_{R_{2}}\right)$ is a curve. In the linear approximation, we have

$$
\left(n_{1} a_{11}+n_{2} a_{12}\right) \Delta J_{R_{1}}+\left(n_{1} a_{12}+n_{2} a_{22}\right) \Delta J_{R_{2}}+\ldots=0
$$

The above equation defines a 'resonant line', which is the local linear approximation to a 'resonant curve'. All resonant lines (or curves) pass through the point $\left(J_{R_{1,0}}, J_{R_{2,0}}\right)$, which, therefore, belongs to the resonant junction defined by the wavevectors $k^{(1)}, k^{(2)}$. To each resonant curve we can associate a resonant strip in action space whose width is proportional to the separatrix width for that resonance. If, for a single pair of integers $\left(n_{1}, n_{2}\right)$, we only isolate the resonant terms $g_{ \pm n_{1}, \pm n_{2}} e^{ \pm i\left(n_{1} \phi_{R_{1}}+n_{2} \phi_{R_{2}}\right)}$ in the normal form $Z$ (Eq.(24)), we obtain a simplified resonant normal form $Z_{r e s\left(n_{1}, n_{2}\right)}$ corresponding to the limiting case of a single resonance. In a strict sense, $Z_{\text {res }}$ describes well the dynamics far from the resonant junction. However, it can also be used in order to obtain estimates of the resonance width along the whole resonant curve defined by the integer pair $\left(n_{1}, n_{2}\right)$. To this end, the leading terms of $Z_{r e s\left(n_{1}, n_{2}\right)}$ are (apart from constants):

$$
\begin{aligned}
Z_{\text {res }\left(n_{1}, n_{2}\right)} & =\epsilon^{1 / 2}\left[\frac{1}{2} a_{11} \Delta J_{R_{1}}^{2}+a_{12} \Delta J_{R_{1}} \Delta J_{R_{2}}+\frac{1}{2} a_{22} \Delta J_{R_{2}}^{2}+\ldots\right. \\
& \left.+\left(g_{n_{1}, n_{2}} e^{i\left(n_{1} \phi_{R_{1}}+n_{2} \phi_{R_{2}}\right)}+g_{-n_{1},-n_{2}} e^{-i\left(n_{1} \phi_{R_{1}}+n_{2} \phi_{R_{2}}\right)}\right)\right]+\ldots
\end{aligned}
$$

where the coefficients $g_{ \pm n_{1}, \pm n_{2}}$ satisfy the estimate

$$
\left|g_{n_{1}, n_{2}}\right| \approx A e^{-\left(\left|n_{1}\right|\left|k^{(1)}\right|+\left|n_{2}\right|\left|k^{(2)}\right|\right) \sigma},
$$

due to Eq.(4). After still another transformation $\Delta J_{R_{1}}=n_{1} J_{R}+n_{2} J_{F}, \Delta J_{R_{2}}=n_{2} J_{R}-n_{1} J_{F}$, $\phi_{R}=n_{1} \phi_{R_{1}}+n_{2} \phi_{R_{2}}, J_{F}$ becomes a second integral of motion of $Z_{r e s\left(n_{1}, n_{2}\right)}$, which takes the form

$$
\begin{aligned}
Z_{\text {res }\left(n_{1}, n_{2}\right)} & =\epsilon^{1 / 2}\left[c\left(J_{F}\right)-\frac{1}{2}\left(a_{11} n_{11}^{2}+2 a_{12} n_{1} n_{2}+a_{22} n_{2}^{2}\right)\left(J_{R}-J_{R, 0}\left(J_{F}\right)\right)^{2}\right. \\
& \left.+\left(g_{n_{1}, n_{2}} e^{i \phi_{R}}+g_{-n_{1}, n_{2}} e^{-i \phi_{R}}\right)+\ldots\right]
\end{aligned}
$$

where $c\left(J_{F}\right)$ and $J_{R, 0}\left(J_{F}\right)$ are constants of the Hamiltonian flow of (36). Combining (35) and (36), the separatrix width can be estimated as

$$
\Delta J_{R} \approx \sqrt{\frac{32 A e^{-\left(n_{1}|| k^{(1)}|+| n_{n}|| k^{(2)}\right) \mid \sigma}}{a_{11} n_{11}^{2}+2 a_{12} n_{1} n_{2}+a_{22} n_{2}^{2}}} .
$$

Eq. (37) allows to estimate the width of a resonant strip in the direction normal to a resonant curve on the plane $\left(J_{R_{1}}, J_{R_{2}}\right)$. Using the relations $\Delta\left(\Delta J_{R_{i}}\right)=n_{i} \Delta J_{R}$ (for $\Delta J_{F}=0$ ), this estimate takes the form

$$
\Delta J_{R, \text { width }}=\approx\left(\frac{32 A\left(n_{1}^{2}+n_{2}^{2}\right)}{a_{11} n_{11}^{2}+2 a_{12} n_{1} n_{2}+a_{22} n_{2}^{2}}\right)^{1 / 2} e^{-\frac{1}{2}\left(\left|n_{1}\right|\left|k^{(1)}\right|+\left|n_{2}\right|\left|k^{(2)}\right|\right) \sigma} .
$$




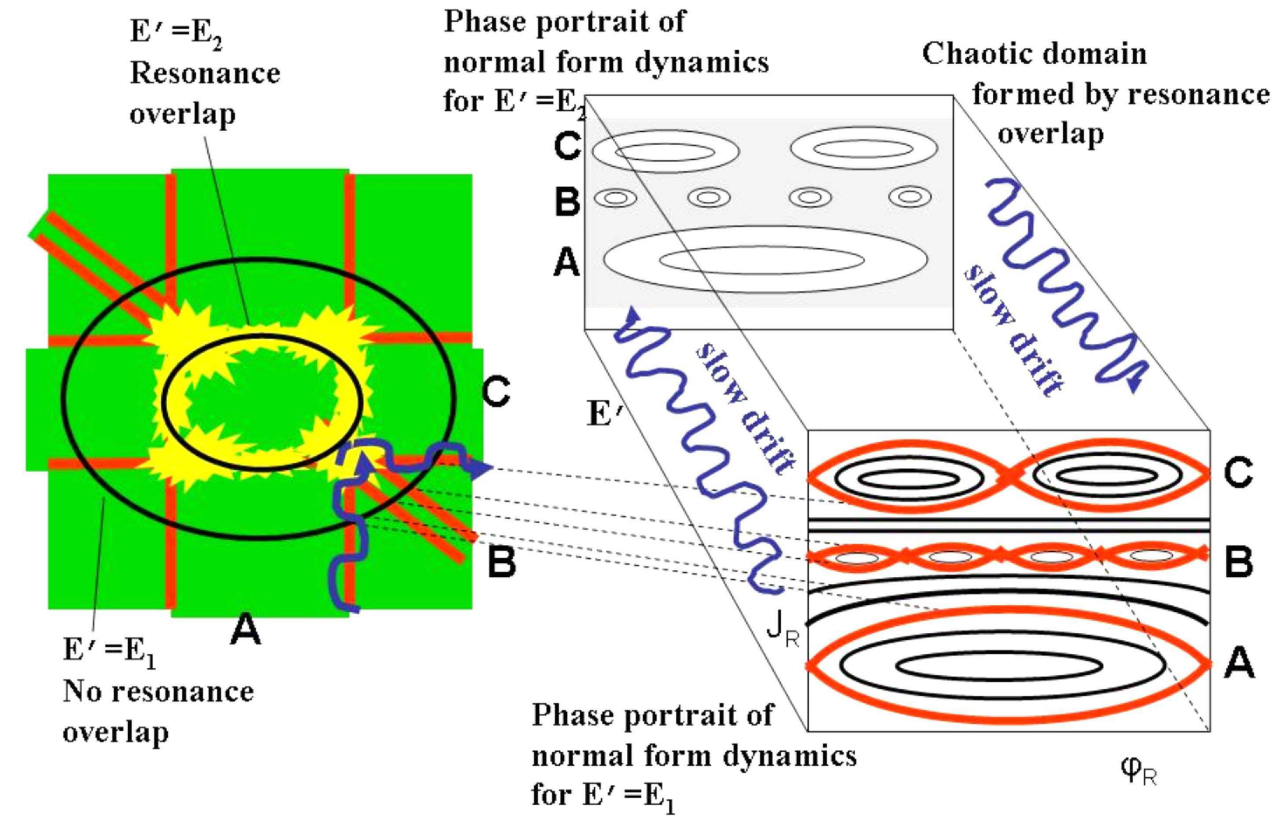

Figure 1: Schematic representation of the normal form and remainder dynamics in a domain of double resonance. Left panel: the resonant structure formed in the action plane of the variables $\left(J_{R_{1}}, J_{R_{2}}\right)$ by the overlapping of various resonant strips whose limits (pairs of parallel red lines) correspond to separatrix-like thin chaotic domains around each resonance. Two constant normal form energy ellipses $E^{\prime}=E_{1}$ and $E^{\prime}=E_{2}$ are also shown. Right: The front and back panels show the phase portraits corresponding to a surface of section (in one of the pairs $\left(\phi_{R_{1}}, J_{R_{1}}\right)$ or $\left(\phi_{R_{2}}, J_{R_{2}}\right)$ ) under the normal form dynamics alone, for the energies $E^{\prime}=E_{1}$ (front panel) and $E^{\prime}=E_{2}$ (back panel). The blue curly arrows in both panels indicate the directions of a possible 'drift' motion (=slow change of the value of $E^{\prime}$ ) due to the influence of the remainder on dynamics. 
The outcome of the analysis so far can be visualized with the help of Figure 1 (schematic). The left panel shows the structure of a doubly-resonant domain in the plane of the resonant action variables $\left(J_{R_{1}}, J_{R_{2}}\right)$. The two bold ellipses correspond to the constant energy condition for two different values of $E^{\prime}$, namely $E^{\prime}=E_{1}$ and $E^{\prime}=E_{2}$ with $E_{1}>E_{2}$. Their common center is the point $\left(J_{R_{1,0}}, J_{R_{2,0}}\right)$ defined in Eq. (32). The three pairs of parallel red lines depict the borders of the separatrix-like thin chaotic layers of three resonances passing through the center. Infinitely many such resonances exist, corresponding to different choices of integer vectors $n \equiv\left(n_{1}, n_{2}\right)$; however, their width decreases as $|n|$ increases, according to Eq. 38). We thus show schematically only three resonances with a relatively low value of $|n|$, named by the letters 'A', 'B' and ' $\mathrm{C}$ '. The blue curly curves indicate a slow drift undergone by the chaotic orbits along the resonance layers, allowing for a transition from one resonance to another. This phenomenon, which will be addressed in detail below, is due to the influence of the remainder terms of the normalized Hamiltonian on dynamics. Here, however, we discuss first the (non-trivial) influence of the normal form terms on dynamics, by considering the Hamiltonian flow under the approximation $H \simeq Z$. Then, the following facts hold:

- For any fixed value of $E^{\prime}$, and a fixed section in the angles, the motion is confined on one ellipse.

- For $E^{\prime}$ large enough $\left(E^{\prime}=E_{1}\right.$, outermost ellipse in the left panel of Fig 1 , the various resonant strips intersect the ellipse $E^{\prime}=E_{1}$ at well distinct arcs, i.e. there is no resonance overlap. The right front panel in Fig 1 shows schematically the expected phase portrait, which can be obtained by evaluating an appropriate Poincaré surface of section, e.g. in the variables $\left(J_{R_{1}}, \phi_{R_{1}}\right)$ or $\left(J_{R_{2}}, \phi_{R_{2}}\right)$. The dashed lines show the correspondence between the limits of various resonant domains depicted in the left and right panels. In particular, the intersection of each resonant strip in the left panel with the ellipse $E^{\prime}=E_{1}$ corresponds to the appearance of an associated island chain in the right panel. The size of islands is given essentially by the separatrix width estimate of Eq.(38). Hence, the size of the islands decreases exponentially with the order of the resonance $n=\left|n_{1}\right|+\left|n_{2}\right|$. However, the main effect to note is that, since all resonant strips are well separated on the ellipse, the thin separatrix-like chaotic layers marking the borders of each of their respective island chains do not overlap. As a result the local chaos around one resonance is isolated from the local chaos around the other resonances. In fact, the normal form dynamics induces the presence of rotational KAM tori which, in this approximation $(H \simeq Z$ ), completely obstruct the communication among the resonances. Note that a detailed study of the dynamics of the above type, induced by the doubly-resonant normal form, was recently presented in [24].

- Far from the domain of resonance overlap, the size of the islands corresponding to each resonance is nearly independent of the energy $E^{\prime}$, as it depends essentially only on the size of the Fourier coefficient of the corresponding harmonics in the Hamiltonian. However, the separation of the islands is reduced as the energy decreases, since this separation is given essentially by the separation between the distinct arcs in Fig 1 at which the various resonances intersect the ellipse corresponding to a fixed energy $E^{\prime}$. As a result, below a critical energy $E_{c}^{\prime}$, significant resonance overlap takes place, leading to the communication of the chaotic layers of the various resonances and an overall increase of chaos. This is shown in the left panel of Fig 1 for an ellipse $E^{\prime}=E_{2}<E_{c}^{\prime}$, with the corresponding phase portrait shown in the right back panel. We note in particular the 'merging' of all three resonant domains one into the other, which produces a large connected chaotic domain surrounding all three island chains (and many other smaller chains, not visible in this scale). 
The value of the critical energy $E_{c}^{\prime}$ marking the onset of large scale resonance overlap can be estimated as follows: Each resonant strip intersects one fixed energy ellipse on one arc segment. Also, Eq. (38) can be replaced by the estimate

$$
\Delta J_{R, w i d t h} \approx \frac{(32 A)^{1 / 2}}{M_{h} k_{1,2}} e^{-\frac{1}{2} n k_{1,2} \sigma}
$$

where $n=\left|n_{1}\right|+\left|n_{2}\right|, k_{1,2}=\left(\left|k^{(1)}\right|+\left|k^{(2)}\right|\right) / 2$, and $M_{h}=\left(\mu_{\min }+\mu_{\max }\right) / 2$, with the constants $\mu_{\min }, \mu_{\max }$ defined as in Eq. (6). The total length $S_{\text {res }}$ of all segments can be now estimated by summing, for all $n$, the estimate (39), namely

$$
S_{r e s} \approx \frac{(32 A)^{1 / 2}}{M_{h} k_{1,2}} \sum_{n=1}^{\infty} e^{-\frac{1}{2} n k_{1,2} \sigma} \approx \frac{(128 A)^{1 / 2}}{M_{h} k_{1,2} \sigma} e^{-\frac{1}{2} k_{1,2} \sigma}
$$

On the other hand, the total circumference of the ellipse for the energy $E^{\prime}$ is estimated as $S_{E^{\prime}}=$ $\pi R\left(E^{\prime}\right)^{2}$ where $R\left(E^{\prime}\right)$ is the geometric mean of the ellipse's major and minor semi-axes. For $R\left(E^{\prime}\right)$ one has the obvious estimate $R\left(E^{\prime}\right) \sim\left(2 E^{\prime} /\left(\epsilon^{1 / 2} M_{h}\right)\right)^{1 / 2}$, whence

$$
S_{E^{\prime}} \sim \frac{2 \pi E^{\prime}}{\epsilon^{1 / 2} M_{h}}
$$

The critical energy $E^{\prime}=E_{c}^{\prime}$ can now be estimated as the value where $S\left(E^{\prime}\right) \approx S_{\text {res }}$, implying that the associated ellipse is fully covered by segments of resonant strips. Thus

$$
E_{c}^{\prime} \approx \frac{32(\epsilon A)^{1 / 2}}{\pi k_{1,2} \sigma} e^{-\frac{1}{2} k_{1,2} \sigma}
$$

Eq. (42) implies that $E_{c}^{\prime}$ is a $O\left(\epsilon^{1 / 2} e^{-\frac{1}{2} k_{1,2} \sigma}\right)$ quantity.

So far, we have neglected the role of the remainder in dynamics. In Fig 1 the drift in action space caused by the remainder is shown schematically by the blue curly curves in both the left and right panels. Their significance is the following: The energy $E=h$ corresponding to the total Hamiltonian $h=Z+R^{\left(r_{\text {opt }}\right)}$ of Eq. (26) is an exactly preserved quantity. Thus, the doubly-resonant normal form energy $E^{\prime}$ as well as $J_{F}$ cannot be preserved exactly, but they are approximate integrals, i.e. they undergo time variations bounded by an $O\left(\left\|R^{\left(r_{\text {opt }}\right)}\right\|\right)$ quantity. In Fig 1, such variations will in general lead to a very slow change of the value of $E^{\prime}$, i.e. a very slow drift of the chaotic orbits from one ellipse to another. We seek to estimate the time required for the remainder to induce a transition between two ellipses with an energy difference of the same order as $E_{c}^{\prime}$, namely

$$
E_{2}^{\prime}-E_{1}^{\prime}=O\left(\epsilon^{1 / 2} e^{-\frac{1}{2} k_{1,2} \sigma}\right)
$$

assuming that this effect can be described as a random walk in the value of $E^{\prime}$ (numerical evidence for this assumption will be provided in section 3). Let $T$ be an average period of the oscillations of the resonant variables. By Eqs. (34) and (35), the estimate $T \sim(\epsilon A)^{-1 / 2} e^{n_{e f f} k_{1,2} \sigma / 2}$ holds, for a constant $n_{\text {eff }} \sim 1$ marking the order of the most important resonances in (34). In consecutive steps, $d E^{\prime}$ can be either positive or negative, while its typical size is $\left|d E^{\prime}\right| \sim\left\|R_{o p t}\right\|$. Then, after $N$ steps of a random walk (in the values of $E^{\prime}$ ), we find an rms spread of these values given by

$$
\Delta E \approx N^{1 / 2}\left\|R_{o p t}\right\|
$$




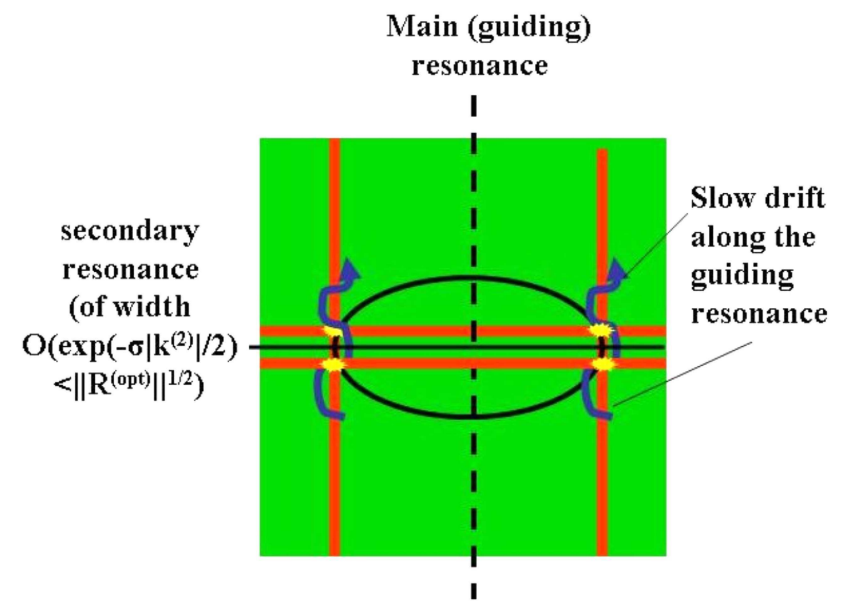

Figure 2: Same as in the left panel of Fig 1 but for a simple resonance. In this case, any other resonance crossing the main (guiding) resonance has an exponentially small width and acts as a 'driving' resonance for diffusion.

Using (43) and (44), the number of steps required for the spread $\Delta E$ to become equal to $E_{2}^{\prime}-E_{1}^{\prime}$ (given by (43) is $N \sim \epsilon e^{-n_{e f f} k_{1,2} \sigma}\left\|R_{o p t}\right\|^{-2}$. The diffusion coefficient can be estimated as

$$
D \sim \frac{\Delta E^{2}}{N T} \sim\left(\epsilon A e^{-n_{e f f} k_{1,2} \sigma}\right)^{1 / 2}\left\|R_{\text {opt }}\right\|^{2}
$$

i.e. the diffusion coefficient scales as the square of the size of the optimal remainder function. This relation is probed by detailed numerical experiments in section 3 .

\subsubsection{Simple resonance}

When $k^{(1)}<K_{\text {opt }}(\epsilon) \leq k^{(2)}, I_{*}$ is simply-resonant with respect to the optimal K-truncation. In this case, the normal form contains terms either independent of the angles, or depending on them via trigonometric terms of the form $\exp \left(i n k^{(1)} \cdot \phi^{\left(r_{o p t}\right)}\right), n \in \mathcal{Z}^{*}$. Using the same notations as in the previous subsection, the transformed Hamiltonian reads:

$$
\begin{aligned}
h\left(J^{\left(r_{o p t}\right)}, \phi^{\left(r_{o p t}\right)}\right) & =Z\left(J^{\left(r_{o p t}\right)}, \phi^{\left(r_{o p t}\right)}\right)+R\left(J^{\left(r_{o p t}\right)}, \phi^{\left(r_{o p t}\right)}\right) \\
& =\omega_{*} \cdot J^{\left(r_{o p t}\right)}+\epsilon^{1 / 2} \sum_{i=1}^{3} \sum_{j=1}^{3} \frac{1}{2} M_{i j *} J_{i}^{\left(r_{o p t}\right)} J_{j}^{\left(r_{o p t}\right)}+\ldots \\
& +\epsilon^{1 / 2} \sum_{n \in \mathcal{Z}^{*}} g_{n}\left(J^{\left(r_{o p t}\right)}\right) \exp \left(i\left(n k^{(1)} \cdot \phi^{\left(r_{o p t}\right)}\right)+\ldots\right. \\
& +R\left(J^{\left(r_{o p t}\right)}, \phi^{\left(r_{o p t}\right)}\right)
\end{aligned}
$$

Repeating all steps as in the case of double resonance leads to the normal form

$$
\begin{aligned}
Z_{\text {res }} & =\frac{1}{2} a_{11} \Delta J_{R_{1}}^{2}+a_{12} \Delta J_{R_{1}} \Delta J_{R_{2}}+\frac{1}{2} a_{22} \Delta J_{R_{2}}^{2}+\ldots \\
& +\epsilon^{1 / 2}\left(g_{n} e^{i n \phi_{R_{1}}}+g_{-n} e^{-i n \phi_{R_{1}}}\right)+\ldots
\end{aligned}
$$


The main difference with respect to the doubly-resonant normal form (34) is that, the angle $\phi_{R_{2}}$ being ignorable, the action $J_{R_{2}}$ (or $\Delta J_{R_{2}}$ ) is an integral of the flow of $Z_{\text {res }}$, in addition to $J_{F}$. Thus, $Z_{\text {res }}$ defines an integrable Hamiltonian. A pair of constant values $J_{F}=c_{1}, \Delta J_{R_{2}}=c_{2}$ defines a straight line

$$
\Delta J_{R_{1}}=-\frac{a_{12}}{a_{11}} c_{2}
$$

which corresponds to the unique resonance $\omega_{R_{1}}\left(J^{\left(r_{\text {opt }}\right)}\right)=k^{(1)} \cdot \omega\left(J^{\left(r_{\text {opt }}\right)}\right)=0$. This will be called 'main resonance' (= the 'guiding resonance' in [9]). In Figure 2] (schematic), the domain of the main resonance is delimited by two vertical thick red lines corresponding to the separatrix-like thin chaotic layers at the boundary of the resonance similarly to Fig 1 Using similar arguments as in the derivation of Eq. (39), the separatrix width can be estimated as

$$
\Delta J_{R, \text { width }} \approx \frac{(32 A)^{1 / 2}}{M_{h}\left|k^{(1)}\right|} e^{-\frac{1}{2}\left|k^{(1)}\right| \sigma}
$$

Under the normal form dynamics, motions are allowed only across the resonance, i.e. in the direction $\Delta J_{R_{2}}=$ const. In Fig 2 this is the horizontal direction. The thin strip delimited by two horizontal red lines corresponds to the resonance with resonant wavevector $k^{(2)}$, which, since $k^{(2)}>K(\epsilon)$, is now of width exponentially small $\left(O\left(\epsilon^{1 / 2} e^{-\sigma\left|k^{(2)}\right| / 2}\right)\right.$. Thus, it will be called a 'secondary' resonance.

In order to estimate the speed of diffusion as a function of the optimal remainder in this case, let us note first that the influence of the remainder on dynamics is to slowly change the value of the two approximate integrals $J_{F}$ and $\Delta J_{R_{2}}$, that would be exactly preserved under the normal form dynamics. In view of Eq. (47), the Hamiltonian (46) can be approximated by

$$
\begin{aligned}
h & \approx\left(m \cdot \omega_{*}\right) J_{F}+\epsilon^{1 / 2}\left[\frac{1}{2} a_{11} \Delta J_{R_{1}}^{2}+a_{12} \Delta J_{R_{1}} \Delta J_{R_{2}}+\frac{1}{2} a_{22} \Delta J_{R_{2}}^{2}+\ldots+2 f_{R_{1}} \cos \left(\phi_{R_{1}}\right)+\ldots\right. \\
& \left.+\sum_{|k| \geq K^{(o p t)}} f_{k *} \exp \left[i k \cdot\left(\kappa_{1} \phi_{R_{1}}+\kappa_{2} \phi_{R_{2}}+\kappa_{3} \phi_{F}\right)\right]+\ldots\right]
\end{aligned}
$$

where i) the (non-integer) vectors $\kappa_{i}, i=1,2,3$ come from the solution of the right Eqs. (25) for the angles $\phi_{i}^{\left(r_{\text {opt }}\right)}$ in terms of the angles $\phi_{R_{1}}, \phi_{R_{2}}$, and $\phi_{F}$, and ii) we approximate all the Fourier coefficients in the remainder series by their constant values $f_{k *}$ at the points $\Delta J_{R_{1}}=\Delta J_{R_{2}}=0$ (we set $f_{R_{1}}=f_{k *}$ for $k=k^{(1)}$ ).

The latter approximation is sufficient for estimates regarding the speed of diffusion. The key remark is that for all the coefficients $f_{k *}$ the bound $\left|f_{k *}\right|<\left\|R_{o p t}\right\|$ holds, while, for the leading Fourier term $\exp \left(i k_{l} \cdot \phi^{\left(r_{o p t}\right)}\right)$ in the remainder we have $\left|f_{k^{*} *}\right| \sim\left\|R_{\text {opt }}\right\|$. In fact, we typically find that the size of the leading term is larger from the size of the remaining terms by several orders of magnitude, since this term contains a repeated product of small divisors of the form $k_{l} \cdot \omega_{*}$ (see Appendix A). Furthermore, using an analysis as in [17], we readily find $\left|k_{l}\right|=(1-d) K_{o p t}$, where $0<d<1$ is a so-called (in [17]) 'delay' constant. We note in passing that the Fourier terms of the form $\exp \left(i k_{l} \cdot \phi^{\left(r_{o p t}\right)}\right)$ are called 'resonant' in [49]. The value of the diffusion coefficient can now be estimated by applying the heuristic theory of Chirikov ([9], see also [11] and [7]) in the Hamiltonian model (50). The estimate

$$
D \sim \frac{\epsilon}{2 \Omega_{G}^{2} T}\left|f_{k^{*} *}\right|^{2} A\left(\left|\kappa_{l}\right|\right)^{2}
$$


holds, where $\Omega_{G}=\epsilon^{1 / 4} f_{R_{1}}^{1 / 2}, T=\ln (32 e / w) / \Omega_{G}$ is an average period of motion within the main resonance separatrix-like thin chaotic layer, of width $w, A$ is the Melnikov function with argument $\left|\kappa_{l}\right|$ (see Appendix B of [21]), the vector $\kappa_{l}$ being defined by the relation $\kappa_{l, 1} \phi_{R_{1}}+\kappa_{l, 2} \phi_{R_{2}}+\kappa_{l, 3} \phi_{F}=$ $k_{l} \cdot \phi^{\left(r_{\text {opt }}\right)}$. The estimate $A\left(\left|\kappa_{l}\right|\right) \sim 8 \pi\left|\kappa_{l}\right| e^{-\pi\left|\kappa_{l}\right| / 2}$ holds. In view of Eq. 25) however, we have that $\left|\kappa_{l}\right|=O\left((1-d) K^{(o p t)} /\left|k^{(1)}\right|\right)$. Since $K_{\text {opt }} \sim \epsilon^{-1 / 4}$ (see Appendix B), and $\left\|R_{\text {opt }}\right\| \sim e^{-\sigma K^{(o p t)}}$, it follows that $A\left(\left|\kappa_{l}\right|\right) \sim \epsilon^{3 / 4} \|\left. R_{o p t}\right|^{b}$, for an exponent $b>0$. Putting these estimates together, we finally arrive at a steeper dependence of the diffusion coefficient $D$ on the optimal remainder $\left\|R_{\text {opt }}\right\|$ in the case of simple resonance than in the case of double resonance, namely:

$$
D \sim \frac{\epsilon}{2 \Omega_{G}^{2} T} \epsilon^{3 / 4}\left\|R_{o p t}\right\|^{2(1+b)}
$$

Regarding now the precise value of $b$, it is hardly tractable to determine this on the basis exclusively of the behavior of the Melnikov integrals discussed above. We note, however, that the quantity $A\left(\kappa_{l}\right)$ yields the size of the 'splitting'S of the separatrix of the main (guiding) resonance due to the effects of the leading term in the remainder function. The relation between the separatrix splitting and the size of the optimal remainder has been examined in [50] and later in [49]. In the latter work, the estimate $S \sim \mu^{1 / 2}$ was predicted and probed by numerical experiments, where $\mu$ (in the notation of [49]) is the effective size of the perturbation to the normal form pendulum dynamics caused by the remainder. Setting thus $\mu \sim\left\|R_{o p t}\right\|$ suggests the scaling $A\left(\kappa_{l}\right) \sim S \sim\left\|R_{\text {opt }}\right\|^{1 / 2}$, whereby the constant $b$ can be estimated as $b \simeq 1 / 2$. Hence (in view of 52.

$$
D \sim\left\|R_{o p t}\right\|^{3}
$$

in simply resonant domains.

Despite the heuristic character of the above derivation, it seems that the value $b \simeq 1 / 2$ is supported by the results of numerical experiments. In particular, in [20] the diffusion coefficient $D$ along a simple resonance was compared directly to the size of the optimal normal form remainder. It was found that $D \propto\left\|R_{\text {opt }}\right\|^{2.98}$, essentially confirming that $p=2(1+b) \simeq 3$. We point out, however, that in [41] a different exponent was found $p \simeq 2.56$ regarding the same resonance as in [20], while it was found that $p=2.1$ in the case of a very low order simple resonance (with $\left|k^{(1)}\right|<K^{\prime}$ ), which is not discussed in our present work. These exponents, on the other hand, depend on the chosen definition of the numerical measure used to estimate both $S$ and $\left\|R_{o p t}\right\|$. Thus, a detailed quantitative comparison of the works cited above is left as on open problem for future study.

\section{Numerical results}

In our numerical work we employ the same Hamiltonian model of three degrees of freedom as in [22, 32, 38, 33]. The Hamiltonian reads:

$$
H=H_{0}+\epsilon H_{1}=\frac{I_{1}^{2}+I_{2}^{2}}{2}+I_{3}+\frac{\epsilon}{4+\cos \phi_{1}+\cos \phi_{2}+\cos \phi_{3}} .
$$

This model has a particularly simple, yet sufficient for our purpose, structure, allowing to probe numerically all steps of the previous section. In particular: 


\subsection{Analyticity and convexity}

The function (53) is polynomial in the action variables, thus it is analytic in any complex extension of $\mathcal{I}=\mathcal{R}^{3}$. On the other hand, the domain of analyticity in the angle variables was examined in [20]. It was found that analyticity can be established in a set $\operatorname{Re}\left(\phi_{i}\right) \in \mathcal{T}, \operatorname{Im}\left(\phi_{i}\right)<\sigma$, $i=1,2,3$, for a positive constant $\sigma$ estimated semi-analytically as $\sigma \simeq 0.82$. Accordingly, the coefficients $h_{k}$ of the Fourier development

$$
\frac{1}{4+\cos \phi_{1}+\cos \phi_{2}+\cos \phi_{3}}=\sum_{k_{1}=-\infty}^{\infty} \sum_{k_{2}=-\infty}^{\infty} \sum_{k_{3}=-\infty}^{\infty} h_{k} \exp (i k \cdot \phi)
$$

where $k \equiv\left(k_{1}, k_{2}, k_{3}\right), \phi \equiv\left(\phi_{1}, \phi_{2}, \phi_{3}\right)$, decay exponentially. The distance of the nearest singularity, with respect to each of the angles $\phi_{i}$, from the real axis is given by the solution of $\cos \phi=-4 / 3$, or $\phi=\pi+0.795365 i$. Thus, the following bound holds:

$$
\left|h_{k}\right| \leq A \exp (-|k| \sigma), \quad A \simeq 0.05, \sigma=0.795365 .
$$

As regards convexity, for all $I_{*} \in \mathcal{I}$ the matrix $M_{*}$ has a particularly simple structure, since we have $M_{11 *}=M_{22 *}=1$, and $M_{i j *}=0$ for all other $i, j$. Thus there are two positive eigenvalues equal to unity and one equal to zero, while $\mu_{\min }=\mu_{\max }=1$.

The constant energy condition $E=\left(I_{1}^{2}+I_{2}^{2}\right) / 2+I_{3}$ defines a paraboloid in the action space. The resonant manifolds are planes, since $\omega_{1}=I_{1}, \omega_{2}=I_{2}, \omega_{3}=1$, whereby the resonance conditions

$$
k_{1} \omega_{1}+k_{2} \omega_{2}+k_{3} \omega_{3}=k_{1} I_{1}+k_{2} I_{2}+k_{3}=0
$$

for all $k \equiv\left(k_{1}, k_{2}, k_{3}\right)$ define planes normal to the $\left(I_{1}, I_{2}\right)$ plane. It follows that, when projected to the $\left(I_{1}, I_{2}\right)$ plane, the intersections of all resonant manifolds with a surface of constant energy of the unperturbed problem yield a set of straight lines. This greatly facilitates the numerical study, since all diffusing orbits in the perturbed problem follow piecewise straight paths nearly parallel to one or more resonant lines of the unperturbed problem, while the orbits can only change direction by approaching close to resonance junctions. Examples of diffusion of this type along a simple resonance where studied in [38], while the case of consecutive encounters with doubly-resonant domains was examined in a mapping model [33] variant of the Hamiltonian model (53).

\subsection{Normal form construction and optimal remainder}

The connection between the size of the optimal remainder $\left\|R_{\text {opt }}\right\|$ and the diffusion coefficient $D$ in a case of simple resonance was the main subject of a previous study [20]. Following the same terminology and notations as in section 2 above, the point $I_{*}$ in the normal form construction in [20] was chosen as $\left(I_{1 *}, I_{2 *}, I_{3 *}\right)=(0.31,0.155,1)$. For this point we have (viz. Eq. (8) ) $k^{(1)}=(1,-2,0), k^{(2)}=(100,0,-31), m=(31,155,100)$. The optimal truncation order in all calculations of [20] varied from $K_{\text {opt }}(\epsilon)=18$ to $K_{\text {opt }}(\epsilon)=39$ (depending on the value of $\epsilon$ in the range considered). Thus, in all cases we have $\left|k^{(1)}\right|<K_{\text {opt }}(\epsilon)<\left|k^{(2)}\right|$, that is the so-chosen point $I_{*}$ was found to be simply resonant with respect to the optimal K-truncation. Following Fig.5 of [20] it was then found by numerical fitting that the diffusion coefficient $D$ scales with the optimal remainder as $D \propto\left\|R_{\text {opt }}\right\|^{2.98}$. A theoretical justification for this 'steepening' of the power-law with respect to the exponent $p \simeq 2$ holding in double resonances was given in subsection 2.3.2. 
In order to probe now the dependence of $D$ on $\left\|R_{\text {opt }}\right\|$ in the case of a double resonance, in the sequel we focus our numerical study on a different point of $\mathcal{D}$, namely $\left(I_{1 *}, I_{2 *}, I_{3 *}\right)=(0.4,0.2,1)$. The basic resonant wavevectors are

$$
k^{(1)}=(1,-2,0), \quad k^{(2)}=(2,1,-1), \quad \text { implying } m=(2,1,5) .
$$

The Hamiltonian normalization is carried out as exposed in subsection 2.2. The interval of values of $\epsilon$ considered is $0.001 \leq \epsilon \leq 0.02$ which, according to [38] is below the critical value for the onset of the 'Nekhoroshev regime' $\left(\epsilon_{c} \simeq 0.03\right)$. Furthermore, it will be shown below that for all values of $\epsilon$ in the above interval the optimal Fourier-truncation order $K_{o p t}$ turns to be much larger than $K=4$. On the other hand, for the basic wavevectors we have $\left|k^{(1)}\right|=3,\left|k^{(2)}\right|=4$. Thus, for all considered values of $\epsilon$ one has $\left|k^{(1)}\right|<\left|k^{(2)}\right|<K_{\text {opt }}(\epsilon)$, that is, the point $I_{*}$ is doubly resonant with respect to any of the optimal $\mathrm{K}$-truncations considered in the sequel.

Due to Eq. 12), the constant $K^{\prime}$ in terms of which book-keeping is implemented changes with $\epsilon$. However, one notices that, because of the logarithmic dependence of $K^{\prime}$ on $\epsilon$, in the largest part of the interval $0.001 \leq \epsilon \leq 0.02$, where we focus, one has a constant value $K^{\prime}=3$, while one has $K^{\prime}=2$ only close to the upper limit $\epsilon=0.02$ and $K^{\prime}=4$ close to the lower limit $\epsilon=0.001$. For simplicity, we thus fixed the value of $K^{\prime}$ as $K^{\prime}=3$ in all normal form computations. Doing so, computer memory limitations restrict all computed expansions to a maximum order $r_{\max }=17$ in the book-keeping parameter $\lambda$, or maximum order $|k|_{\max }=17 K^{\prime}-1=50$ in Fourier space. In fact, for $\epsilon>0.005$ we perform at most 14 normalization steps, so that the remainder contains terms of at least three consecutive orders in $\lambda$, namely $r=15,16$ and 17. As explained below, this allows us to perform some numerical tests regarding the convergence of the remainder series when the optimal normalization order is as high as $r_{\text {opt }}=14$ (or $K_{\text {opt }}=42$ ). On the other hand, for $\epsilon \leq 0.005$ we allow for one more normalization step $(r=15)$ in order to get as close as possible to the optimal order, which, as shown below for $\epsilon<0.004$ is larger than 14 . Thus, for the calculation of the corresponding remainder value at this order $(r=15)$ we necessarily have to rely on the sum of only two rather than three or more consecutive terms.

Writing the truncated (at order 17) remainder function as:

$$
R^{(r)}\left(J^{(r)}, \phi^{(r)}\right)_{\leq 17}=\sum_{s=r+1}^{17} R_{s}^{(r)}\left(J^{(r)}, \phi^{(r)}\right)
$$

where $R_{s}^{(r)}\left(J^{(r)}, \phi^{(r)}\right)$ are the terms of order $s$ in the book-keeping parameter $\lambda$ allows us to probe numerically the convergence of the remainder function within any chosen domain $\mathcal{W}_{I_{*}, B}$ in action space. To this end, at any normalization order $r$, let us consider a disk $\left(J_{1}^{(r)}\right)^{2}+\left(J_{2}^{(r)}\right)^{2} \leq \rho^{2}$ in the space of the transformed action variables (we neglect the action $I_{3}$ which, in the particular case of the Hamiltonian (53), is dummy, i.e. it does not appear in any higher order term of either the normal form or the remainder). This is a deformed disk also in the old canonical variables $J_{1}, J_{2}$, limited by a boundary given approximately by $\epsilon\left(J_{1}^{2}+J_{2}^{2}\right)=\left(I_{1}-I_{1 *}\right)^{2}+\left(I_{2}-I_{2 *}\right)^{2} \simeq \epsilon \rho^{2}$ (cf. the action re-scaling given by Eq.(10)). For the Hamiltonian (53) one can readily check that all the terms in $R_{s}^{(r)}\left(J^{(r)}, \phi^{(r)}\right)$ are trigonometric polynomials of maximum degree $K^{\prime} s-1=3 s-1$ whose coefficients are polynomial of maximum degree $s-1$ in the actions, namely

$$
R_{s}^{(r)}\left(J^{(r)}, \phi^{(r)}\right)=\sum_{|k|=0}^{3 s-1}\left(\sum_{q=0}^{s-1} \sum_{l=0}^{q} R_{k, l, q-l}\left(J_{1}^{(r)}\right)^{l}\left(J_{2}^{(r)}\right)^{q-l}\right) \exp \left(i k \cdot \phi^{(r)}\right)
$$


Furthermore, in the disk $\mathcal{W}_{I_{*}, \epsilon^{1 / 2} \rho}$ the obvious bound

$$
\sup _{\mathcal{W}_{I_{*}, \epsilon^{1 / 2} \rho}}\left|\left(J_{1}^{(r)}\right)^{l}\left(J_{2}^{(r)}\right)^{q-l}\right|=\left(\frac{l^{l / 2}(q-l)^{(q-l) / 2}}{q^{q / 2}}\right) \rho^{q}
$$

holds. We thus define the norm

$$
\left\|R_{s}^{(r)}\left(J^{(r)}, \phi^{(r)}\right)\right\|_{W_{l_{k, \epsilon^{1 / 2} \rho}}}=\sum_{|k|=0}^{3 s-1} \sum_{q=0}^{s-1} \sum_{l=0}^{q}\left|R_{k, l, q-l}\right|\left(\frac{l^{l / 2}(q-l)^{(q-l) / 2}}{q^{q / 2}}\right) \rho^{q}
$$

in view of which a numerical estimate of the size of the remainder within $\mathcal{W}_{I_{*}, \rho}$ can be obtained. In fact, by calculating the truncated sums

$$
\left\|R^{(r)}\left(J^{(r)}, \phi^{(r)}\right)\right\|_{\leq p, W_{I_{*}, \epsilon^{1 / 2} \rho}}=\sum_{s=r+1}^{p}\left\|R_{s}^{(r)}\left(J^{(r)}, \phi^{(r)}\right)\right\|_{W_{I *, \epsilon / 2} \rho}
$$

for any fixed choice of $\rho$, where $p$ takes all values $p=r+1, r+2, \ldots, 17$, we can have a clear numerical indication of whether the remainder function was calculated up to a sufficiently high order for convergence to have been practically reached.

The maximum value of $\rho$ for which the series $\left\|R^{(r)}\left(J^{(r)}, \phi^{(r)}\right)\right\|_{\leq \infty, W_{I_{*}, \epsilon^{1 / 2} \rho}}$ converges absolutely sets the size of the doubly-resonant domain $B=\epsilon^{1 / 2} \rho_{\max }$ (in non-scaled variables) where the normal form calculations are valid. In practice, we are interested in the diffusion of orbits with initial conditions inside this domain. In particular, in subsection 3.2 we will consider orbits starting on the circle $\rho_{0}=0.27$. All our numerical orbits are studied up to a time in which their distance from the center of the double resonance changes significantly less than $\Delta \rho=10^{-1}$ (see below). Variations of this order at maximum are found when we measure $\rho$ either in the original canonical action variables or in the variables after the optimal canonical transformation. Thus, for all the orbits we can set a safe outer boundary $\rho<\rho_{b}=0.4$ within which they are well confined. We then verify numerically that this domain belongs to the analyticity domain of the various transformations employed in the form of series (of the new variables in terms of the old variables or vice versa). This check is made by finding whether the Fourier coefficients of the series exhibit an exponential decay. An example is given in Fig 3 , We consider the Fourier series yielding the new transformed canonical action $J_{1}^{(r)}$ as a function of the old canonical variables, for $\epsilon=0.01$, at the normalization orders $r=4,8$ and 11 . Writing this as a series

$$
J_{1}^{(r)}=J_{1}+\sum_{k} \sum_{s_{1}, s_{2}=0}^{s_{\max }(k)} g_{k, s_{1}, s_{2}}^{(r)} J_{1}^{s_{1}} J_{2}^{s_{2}} \exp (i k \cdot \phi)
$$

we define the coefficients

$$
G_{|k|}^{(r)}=\sum_{k_{1}, k_{2},\left|k_{1}\right|+\left|k_{2}\right|=|k|} \sum_{s_{1}, s_{2}=0}^{s_{\max }(k)}\left|g_{k, s_{1}, s_{2}}\right| \frac{s_{1}^{s_{1} / 2} s_{2}^{s_{2} / 2}}{\left(s_{1}+s_{2}\right)^{\left(s_{1}+s_{2}\right) / 2}} \rho_{b}^{s_{1}+s_{2}}
$$

Figure 3 shows the coefficients $G_{\mid} k^{(r)}$ for $\epsilon=0.01, \rho_{b}=0.4$, and $r=4,8$ and 11 . We observe that all three curves exhibit a tail showing exponential decay of the Fourier coefficients. However, it is remarkable that the asymptotic exponential slope seems to change only marginally. Instead, 


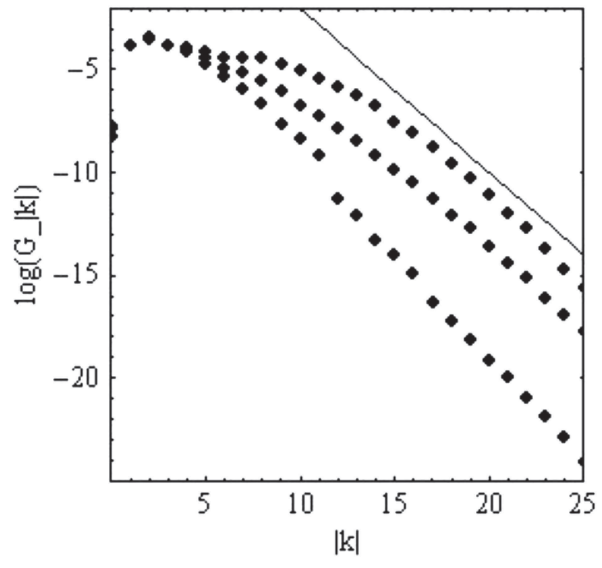

Figure 3: The logarithm of the quantity $G_{|k|}^{(r)}$ (see text) as a function of the Fourier order $|k|$, for $\epsilon=0.01$, at the normalization orders $r=4, r=8$, and $r=11$ (lower, middle and upper set of points respectively). All three curves exhibit an exponential decay for large $|k|$, with nearly the same asymptotic law. The straight line has inclination $\sigma=-0.8$.

the main change, as $r$ increases, regards that formation of a 'plateau' of Fourier coefficients of nearly constant size formed for small $|k|$. Namely, the width of the plateau increases as $r$ increases. It is remarkable that the asymptotic tail laws for all $r$ appear to follow an exponential decay with the same constant $\sigma \simeq 0.8$, i.e. with nearly the same value as the constant appearing in the analyticity condition of the original Hamiltonian (cf. Eq.(55)). This effect shows that, while in the usual proofs of the Nekhoroshev theorem one requires a reduction of the analyticity domain at every normalization step, i.e. one considers bounds of the form $G_{\mid} k^{(r)} \leq A^{(r)} e^{-\sigma_{r}|k|}$ with $\sigma_{r}<\sigma_{r-1}<\ldots<\sigma_{1}$, in practice the dependence of the coefficients $G_{|k|}^{(r)}$ on $|k|$ is more complicated than a simple exponential decay law. In fact, the constants $\sigma_{r}$ reflect an average exponential slope that compensates between the plateau, for small $|k|$, and the exponential tail, for large $|k|$. Namely, as the width of the plateau increases with $r$, one obtains smaller and smaller values of the average exponential decay constant $\sigma_{r}$.

Fig 4 a shows now an example of the behavior of the truncated remainder function for $\rho=\rho_{0}$ and $\epsilon=0.01$. The upper curve shows the value of $\left\|R^{(r)}\left(J^{(r)}, \phi^{(r)}\right)\right\|_{\leq p, W_{I+, \epsilon^{1 / 2} \rho_{0}}}$ at the normalization order $r=6$ as a function of $p$ for $p=7, \ldots 17$. Clearly, after $p=9$ the cumulative sum (62) shows no further substantial variation, which indicates that the remainder series converges after three consecutive terms $p=7,8$ and 9 (this is verified also by computing numerically a convergence criterion like d'Alembert's criterion). The lower and middle curves show now the same effect for the normalization orders $r=11$ and $r=14$ respectively. Note that the three consecutive truncation orders $p=15,16$ and 17 allowed for the computation of the remainder at the normalization order $r=14$ are essentially sufficient to demonstrate the convergence of the remainder. Hence, $\left\|R^{(r)}\left(J^{(r)}, \phi^{(r)}\right)\right\|_{\leq 17, W_{I_{*}, \epsilon^{1 / 2} \rho_{0}}}$ represents a good numerical estimator of the value of the remainder series for normalization orders up to $r=14$. However, the main effect to note is that the estimated remainder value $\left\|R^{(r)}\left(J^{(r)}, \phi^{(r)}\right)\right\|_{\leq 17, W_{I_{*}, \epsilon^{1 / 2} \rho_{0}}}$ found for $r=14$ is larger than the one for $r=11$, implying that the optimal normalization order $r_{\text {opt }}$ is below $r=14$. Fig $4 \mathrm{~b}$ shows, precisely, the asymptotic character of the above normalization, showing $\left\|R^{(r)}\left(J^{(r)}, \phi^{(r)}\right)\right\|_{\leq 17, W_{I_{*}, \epsilon^{1 / 2} \rho_{0}}}$ against the 

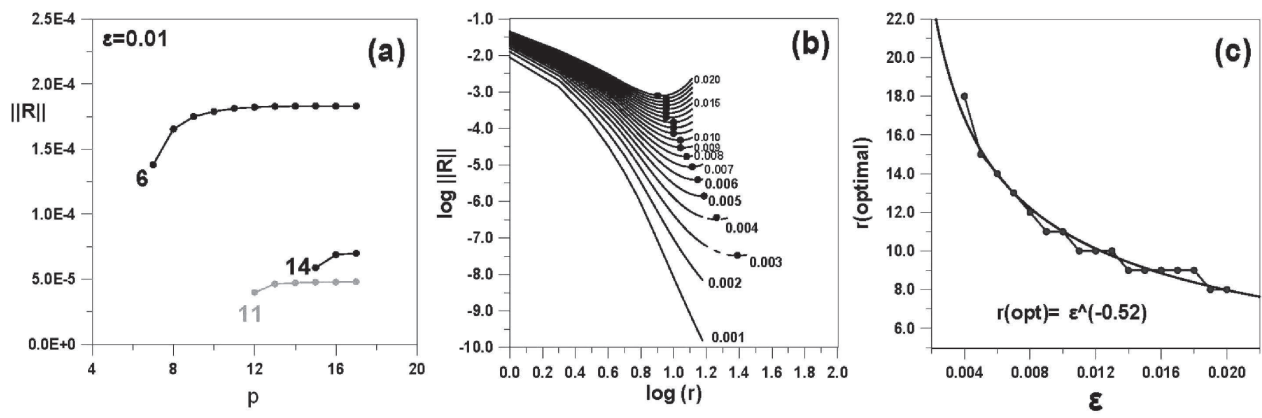

Figure 4: (a) The value of the remainder norm $\left\|R^{(r)}\right\|_{\leq p, W_{I *, \epsilon} / 2 \rho_{0}}$ as a function of the truncation order $p$ when $\epsilon=0.01$, $\rho_{0}=0.27$, and the normalization orders are $r=6$ (upper curve), $r=11$ (lower curve) and $r=14$ (middle curve). (b) The value of $\left\|R^{(r)}\right\|_{\leq 17, W_{I_{*}, \rho_{0}}}$ as a function of $r$ for different values of $\epsilon$. For $\epsilon=0.004$ and $\epsilon=0.003$, the dashed curves after the order $r=15$ are found by quadratic extrapolation. No attempt to extrapolate was made for $\epsilon=0.002$ and $\epsilon=0.001$. (c) The optimal normalization order $r_{o p t}$ as a function of $\epsilon$ together with a power-law best fitting curve.

normalization order $r$ for various values of $\epsilon$ as indicated in the figure. For all values down to $\epsilon=0.005$ we now observe the asymptotic behavior, namely the size of the remainder initially decreases as $r$ increases, giving the impression that the normalization might be a convergent procedure. However, this trend is reversed after an optimal order $r_{o p t}$, where the remainder reaches its minimum value, while, for $r>r_{\text {opt }}$ the remainder increases with $r$ and eventually goes to infinity. We also observe that for $\epsilon \leq 0.004$ the optimal order is beyond $r=15$. However, for $\epsilon=0.004$ and $\epsilon=0.003$, the computed remainder values are close to the minimum. The dashed extensions of the numerical curves shown in Fig $4 \mathrm{~b}$ correspond to an extrapolation obtained by quadratic fitting of the available numerical points near the corresponding minima. Using this extrapolation, we obtain an estimate of the optimal remainder size for the values $\epsilon=0.004$ and $\epsilon=0.003$, that will be used in some calculations below. On the other hand, for $\epsilon=0.002$ and $\epsilon=0.001$, even using the extrapolation we find that the optimal normalization is beyond any reliable possibility to estimate given our computing limitations.

As discussed above, the estimate $r_{\text {opt }} \propto 1 / \epsilon^{1 / 2}$ holds [54], i.e. $r_{o p t}$ is expected to be a decreasing function of $\epsilon$. Fig 4 , shows the numerical estimate for $r_{\text {opt }}$ as a function of $\epsilon$ from the points of minima of Fig $4 \mathrm{~b}$. The blue curve is a power-law fitting, yielding the exponent 0.52 , i.e. very close to the one predicted by theory.

Since the value $\left\|R_{\text {opt }}\right\|=\left\|R^{\left(r_{\text {opt }}\right)}\left(\boldsymbol{J}^{(r)}, \phi^{(r)}\right)\right\|_{\leq 17, W_{I_{*, \epsilon} / 2 \rho_{0}}}$ depends on $\epsilon$, from the above procedure we obtain numerically pairs of values $\left(\epsilon,\left\|R_{o p t}\right\|(\epsilon)\right)$. In subsection 3.4 below, we will numerically calculate the value of the diffusion coefficient $D$ for each one of the selected values of $\epsilon$, thus allowing for a probe of the dependence of $D$ on $\left\|R_{\text {opt }}\right\|$ and a comparison with the results of subsection 2.3.1.

\subsection{Resonant structure}

The resonant structure in the action space (around $I_{*}$ ) can be visualized by employing the method of the FLI map as in [22, 38]. We recall that the Fast Lyapunov indicator (FLI) is a numerical indicator of chaos, defined for one orbit by

$$
F L I=\log _{25}|\xi(t)|
$$



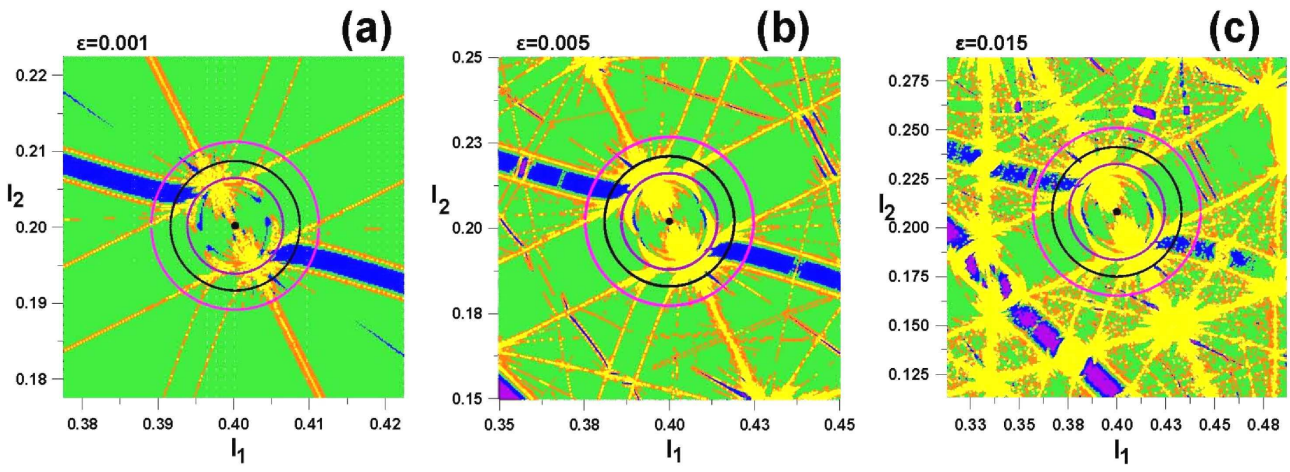

Figure 5: FLI map in the action space (surface of section $\left(I_{1}, I_{2}\right)$ of the Hamiltonian 53 for $\phi_{3}=0,\left|\phi_{1}\right|+\left|\phi_{2}\right|=0$, around the doubly-resonant point $\left(I_{1 *}, I_{2 *}\right)=(0.4,0.2)$ for (a) $\epsilon=0.001$, (b) $\epsilon=0.005$, (c) $\epsilon=0.015$. The color scale represents the computed value of the FLI (see text) in the intervals $2 \leq F L I<3$ (magenta, most ordered), $3 \leq F L I<3.5$ (blue), $3.5 \leq F L I<4$ (green), $4 \leq F L I<5$ (orange), $5 \leq F L I$ (yellow, most chaotic).

where $\xi(t)$ is a deviation vector, i.e. in our case $\xi(t) \equiv\left(\Delta \phi_{1}(t), \Delta \phi_{2}(t), \Delta \phi_{3}(t), \Delta I_{1}(t), \Delta I_{2}(t), \Delta I_{3}(t)\right)$ found after solving the variational equations of motion up to the time $t$ from some initial conditions $\xi(0)$. By properly choosing a threshold value $F L I_{0} \sim \log _{10} t$, orbits with $F L I<F L I_{0}$ are characterized as regular, and those with $F L I>F L I_{0}$ as chaotic. Furthermore, a convenient use of the FLI in the visualization of the Arnold web is found by producing FLI color maps [22]. Considering a grid of initial conditions in the action space, we assign to each initial condition a color corresponding to the FLI value found for the resulting orbit integrated up to a sufficiently long time (of the order $100-1000$ periods). This allows for illustrating the resonant structure in action space, as shown in Fig 5, which is an FLI map in an action domain including our chosen doubly-resonant point $\left(I_{1 *}, I_{2 *}\right)=(0.4,0.2)$ for three different values of $\epsilon$. In all three panels, there are resonances projecting on $\left(I_{1}, I_{2}\right)$ as single yellow or orange thick lines, while other resonances project as strips with a green or blue interior zone delimited by pairs of nearly parallel yellow or red lines. As explained in [20], this difference is only due to the particular choice of surface of section ( $\phi_{3}=0,\left|\phi_{1}\right|+\left|\phi_{2}\right| \leq 0.1$, similar to [38]). Namely, the yellow lines marking all resonances represent the intersection of the thin separatrix-like chaotic layers formed around each resonance with the chosen surface of section. This produces a pair of nearly parallel yellow or orange lines for any resonance (of the form $k \cdot \omega=0$ ) whose leading Fourier coefficient $h_{k}$ of the resonant term $\exp (i k \cdot \phi)$ in the original Hamiltonian expansion has a negative real part, while it produces a single yellow or orange thick line if $\operatorname{Re}\left(h_{k}\right)$ is positive. In the latter case, the domain of regular orbits inside the resonance has no projection on the chosen surface of section, while in the former case it projects as a strip of green or blue color.

When $\epsilon=0.001$ ( $\mathrm{Fig} 5 \mathrm{5}$ ), we easily distinguish four main resonances passing through $\left(I_{1 *}, I_{2 *}\right)=(0.4,0.2)$. The biggest resonant domain (green, from bottom left to top right) corresponds to the resonance $\omega_{1}-2 \omega_{2}=0$, whose corresponding wave-vector is the basic resonant wavevector $k^{(1)}$. Similarly, the single yellow-red thick line going from bottom right to top left is the resonance $2 \omega_{1}+\omega_{2}-\omega_{3}=0$, whose corresponding (also basic) wavevector is $k^{(2)}$. We also clearly distinguish two resonances of order $|k|=5$, namely $\omega_{1}+3 \omega_{2}-\omega_{3}=0$ (blue), and $3 \omega_{1}-\omega_{2}-\omega_{3}=0$ (green). Many other higher order resonances cross the central doublyresonant point $\left(I_{1 *}, I_{2 *}\right)=(0.4,0.2)$, denoted hereafter by $\mathrm{O}$, but they are not so visible in the 
scale of Fig 5 .

The resonant strips of all previous resonances join each other forming a domain of double resonance around $\mathrm{O}$. The extent of this domain can be determined roughly by drawing concentric circles around the point $\mathrm{O}$. Such circles correspond to nearly constant normal form energy values, as can be seen by noting that, for the particular Hamiltonian function (53), the coefficients $a_{i j}$ of Eq. (34) have the values $a_{11}=5 a_{22}=5$, and $a_{12}=a_{21}=0$. Applying Eqs.(29) 31|32) for the particular resonant wavevectors given by (57), the doubly-resonant normal form of the Hamiltonian (53) expressed in resonant variables takes the form

$$
Z=c_{*}+6 J_{F}+\frac{5 \epsilon^{1 / 2}}{2}\left(J_{R_{1}}^{2}+\left(J_{R_{2}}+J_{F}\right)^{2}\right)+O(\epsilon)
$$

where the $O(\epsilon)$ terms are trigonometric polynomials of the resonant angles $\phi_{R_{1}}=\phi_{1}-2 \phi_{2}$, $\phi_{R_{2}}=2 \phi_{1}+\phi_{2}-\phi_{3}$, while $c_{*}=\epsilon^{1 / 2} 0.28186 \ldots$ is a constant which appears only in the numerical values of the quantity

$$
E_{Z}=Z-6 J_{F}
$$

called, hereafter, the normal form energy ( $E_{Z}$ differs from the quantity $E^{\prime}$ defined in Eq. (30) only by the constant $c_{*}$ ). We note that the estimate $O(\epsilon)$ for the trigonometric terms in $Z$ follows from the estimate (35) for the size of the corresponding Fourier coefficients, taking into account that $e^{-\sigma\left|k^{(1)}\right|} \sim e^{-\sigma\left|k^{(2)}\right|} \sim \epsilon^{1 / 2}$, according to Eq.(12). Since the angle $\phi_{F}$ is ignorable in the hamiltonian (66), $J_{F}$ is an integral of the flow of $Z$. Furthermore, since for the particular choice of Hamiltonian model (53) the action $I_{3}$ is dummy, implying that $I_{3}$ can be assigned any arbitrary value without affecting the dynamical evolution of any other canonical variable, we can always choose the value of $I_{3}$ so that $J_{F}=0$. Then, the normal form energy condition $E_{Z}=$ const implies

$$
\frac{2\left(E_{Z}-c_{*}\right)}{\epsilon^{1 / 2}} \equiv \rho^{2}=5\left(J_{R_{1}}^{2}+J_{R_{2}}^{2}\right)
$$

where $\rho$ is a $O(1)$ quantity. Transforming to the original non-scaled action variables we also find

$$
\left(\epsilon^{1 / 2} \rho\right)^{2} \simeq\left(I_{1}-0.4\right)^{2}+\left(I_{2}-0.2\right)^{2}
$$

whereby $\epsilon^{1 / 2} \rho$ is interpreted as the radius of a circle, around $\mathrm{O}$, corresponding to a constant normal form energy condition. It follows that the set of all possible normal form energy values are represented on the $\left(I_{1}, I_{2}\right)$ plane as a set of concentric circles around $O$. Three such circles are drawn in Fig 5 h, corresponding to $\epsilon=0.001$ and $\rho_{1}=0.31$ (outer circle), $\rho_{2}=0.27$ (middle circle), and $\rho_{3}=0.25$ (inner circle). Their main difference concerns the degree of resonance overlapping in each case. Namely, for a value of $E_{Z}=0.0104$, corresponding to the outer circle $\rho_{1}=0.31$, the main visible resonances of Fig. $(5 \mathrm{k})$ intersect the circle in some arcs only, while the remaining parts of the circle lie in the regular (non-resonant) domain. In the latter parts, the normal form dynamics alone would imply the existence of a set of Kolmogorov-ArnoldMoser invariant tori of large measure. On the contrary, in the inner circle, corresponding to $E_{Z}=0.0099$, all resonances essentially overlap, producing a strongly chaotic domain. The middle circle corresponds to $E_{Z}=0.01007$, which is close to the critical energy below which resonance overlapping dominates the dynamics.

The remaining panels of Fig 5 show what happens when $\epsilon$ is increased by a factor 5 ( $\epsilon=$ 0.005 , Fig. 5b), or 15 ( $\epsilon=0.015$, Fig. 5k) with respect to Fig 5 k. A main feature to notice is that, by increasing $\epsilon$, many more resonances 'show up' in the FLI map. Furthermore, the size 
of all resonant domains grows proportionally to $\epsilon^{1 / 2}$, as verified in Fig $[5$, where by augmenting the scale in panels (b) and (c) by a factor $\sqrt{5}$ and $\sqrt{15}$ respectively with respect to panel (a), the widths of all resonant strips passing through $O$ remain essentially unaltered in all three panels. Thus, the only essential change is the increase of chaos as $\epsilon$ increases. Namely, we see that the chaotic layers delimiting the borders of each resonance become thicker as $\epsilon$ increases. This also increases the resonance overlapping locally, close to the points of resonance crossings.

Focusing, now, on one value $\epsilon=0.008$, Figure 6 shows in detail the implications of normal form dynamics in the two regimes when there is no resonance overlap $\left(E_{Z}=0.0306\right.$, Figs. 6 6,c), or when there is substantial resonance overlap $\left(E_{Z}=0.029\right.$, Figs $\left.6 \mathrm{~b}, \mathrm{~d}\right)$. The upper panels correspond to FLI maps as in Fig.(5). Here, however, instead of the action variables $\left(I_{1}, I_{2}\right)$ we use the resonant re-scaled actions $\left(J_{R_{1}}, J_{R_{2}}\right)$, defined as in Eq.(25), where, for each point in the action space of the original variables we compute the values of the transformed actions $J_{i}^{\left(r_{o p t}\right)}, i=1,2,3$ by the composition of the Lie canonical transformations resulting from the computer-algebraic program calculating the optimal normal form $Z$. Since the same program renders also the algebraic form of $Z$, we use this expression to derive the Hamiltonian equations of motion of the normal form alone, namely $\dot{\phi_{R_{1}}}=\partial Z / \partial J_{R_{1}}, \dot{\dot{\phi}_{R_{2}}}=\partial Z / \partial J_{R_{2}}, J_{R_{1}}=-\partial Z / \partial \phi_{R_{1}}$, $\vec{J}_{R_{2}}=\partial Z / \partial \phi_{R_{2}}, \dot{\phi_{F}}=\partial Z / \partial J_{F}$, while we set $J_{F}=$ const $=0$. For each value of $E_{Z}$, we then compute numerical orbits under the normal form dynamics alone via the previous equations. Finally we plot a convenient surface of section of the normal form flow, taken by the condition $\bmod \left(\phi_{R_{2}}-2 \phi_{R_{1}}, 2 \pi\right)=\bmod \left(5 \phi_{2}-\phi_{3}, 2 \pi\right)=0$. These sections are shown in Figs $6 \mathrm{k}, \mathrm{d}$, for the normal form energy values $E_{Z}=0.0306$ and $E_{Z}=0.0290$ respectively. The corresponding circles, through Eq.(68), are shown in panels (a) and (b), superposed to the color background yielding the FLI map in the resonant action variables for $\epsilon=0.008$. The main feature of this plot is the exact correspondence between the values of $J_{R_{1}}$ where each resonance intersects the circle corresponding to $E_{Z}=$ const in panels (a) and (c), and the projection of these values to thin chaotic layers delimiting the same resonance in the corresponding surface of section. In fact, inside each resonance we have regular orbits corresponding to islands of stability on the surface of section. Furthermore, while at the normal form energy value $E_{Z}=0.0306$ there are many rotational KAM tori separating these resonances, at the value $E_{Z}=0.029$ these tori are destroyed and substantial resonance overlap takes place. This fact leads to the creation of a connected chaotic domain surrounding all main resonances in the surface of section of Fig $6 \mathrm{~d}$. This, in turn, implies that under the normal form dynamics alone no communication is allowed from one resonance to the other for the normal form energy value $E_{Z}=0.0306$ (which in this approximation remains constant in time), while such communication is possible throughout the whole connected chaotic domain for $E_{Z}=0.029$. In fact, the phase portrait of Fig $6 \mathrm{~d}$ renders visually clear that chaos is rather strong in this case. However, as emphasized in section 2, this fact has no consequences regarding the possibility of long excursions in the action space, since all motions in this approximation would be bounded on circles like those of Figs 6 a,b. On the contrary, such excursions are only possible due to the effect of the remainder, which causes the chaotic orbits to slowly 'drift' from circle to circle as the value of $E_{Z}$ changes slowly in time. To this we now turn our attention.

\subsection{Visualization of Arnold diffusion in doubly-resonant normal form variables}

The main effect, of local diffusion within the doubly-resonant domain, can now be demonstrated with the help of Figure 7 . The time evolution of one chaotic orbit is shown in this figure, as the orbit moves within the doubly-resonant domain along some of the main intersecting resonances. In this example as well we take $\epsilon=0.008$ (as in Fig 6 ). 

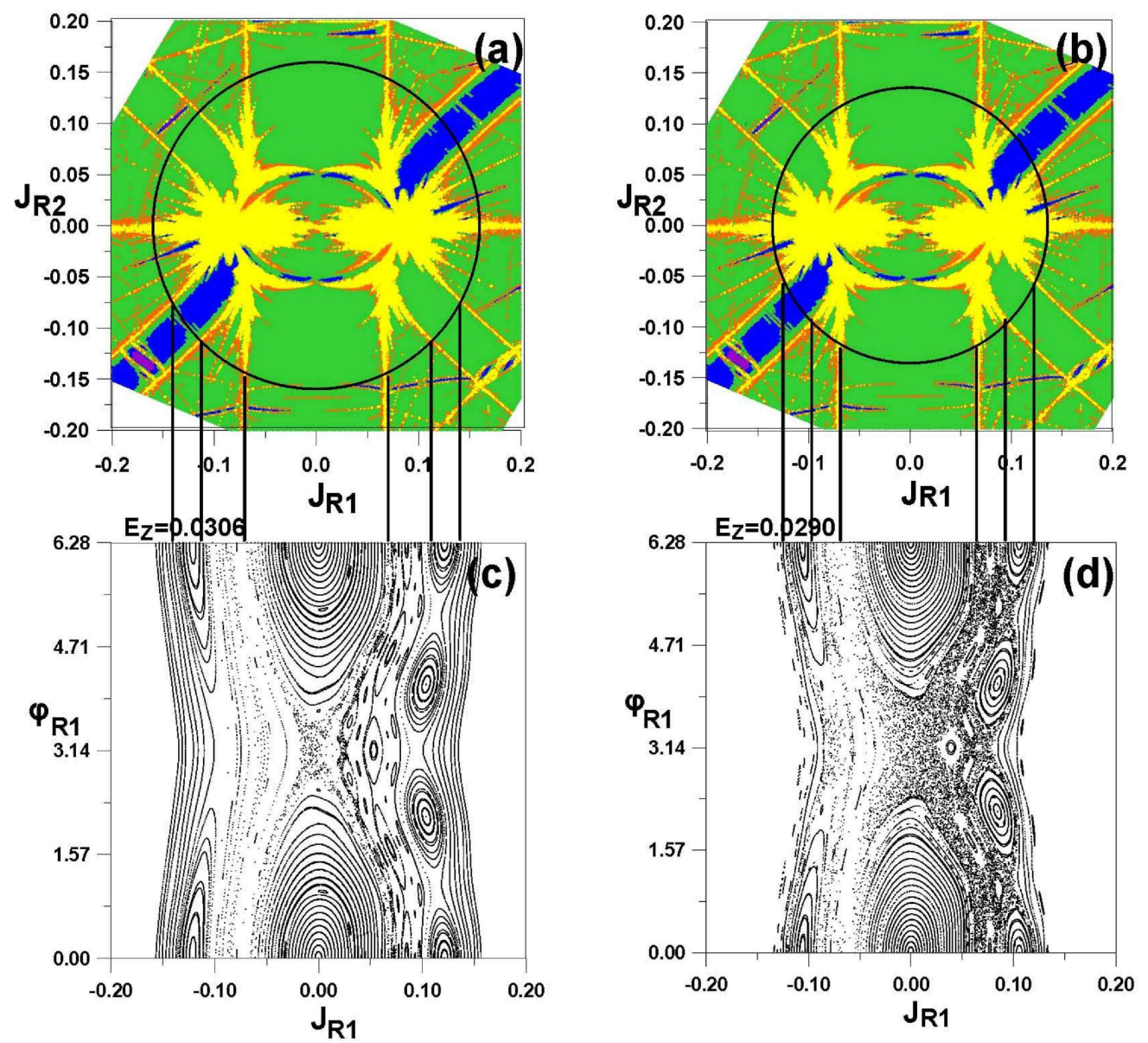

Figure 6: (a) and (b) The FLI map in the plane $\left(J_{R_{1}}, J_{R_{2}}\right)$ defined as in Eqs. 25] for the Hamiltonian [53, for the same surface of section and using the same color scale as in Fig. 5. The circle in (a) corresponds to the constant normal form energy value $E_{Z}=0.0306$ in (a) and $E_{Z}=0.029$ in (b). The phase portraits of the normal form dynamics for the values (c) $E_{Z}=0.0306$ and (d) $E_{Z}=0.029$. The plotted surfaces of sections are $\left(\phi_{R_{1}}, J_{R_{1}}\right)$ whenever the quantity $\phi_{R_{2}}-2 \phi_{R_{1}}=5 \phi_{2}-\phi_{3}$ (where all symbols denote the new canonical coordinates and momenta after the optimal Lie normalization) crosses a multiple value of $2 \pi$. The main resonances are identified as: $\omega_{1}-2 \omega_{2}=0$ (vertical), $2 \omega_{1}+\omega_{2}-\omega_{3}=0$ (horizontal), $\omega_{1}+3 \omega_{2}-\omega_{3}=0$ (bottom left to top right diagonal ), $3 \omega_{1}-\omega_{2}-\omega_{3}=0$ (top left to bottom right diagonal). 

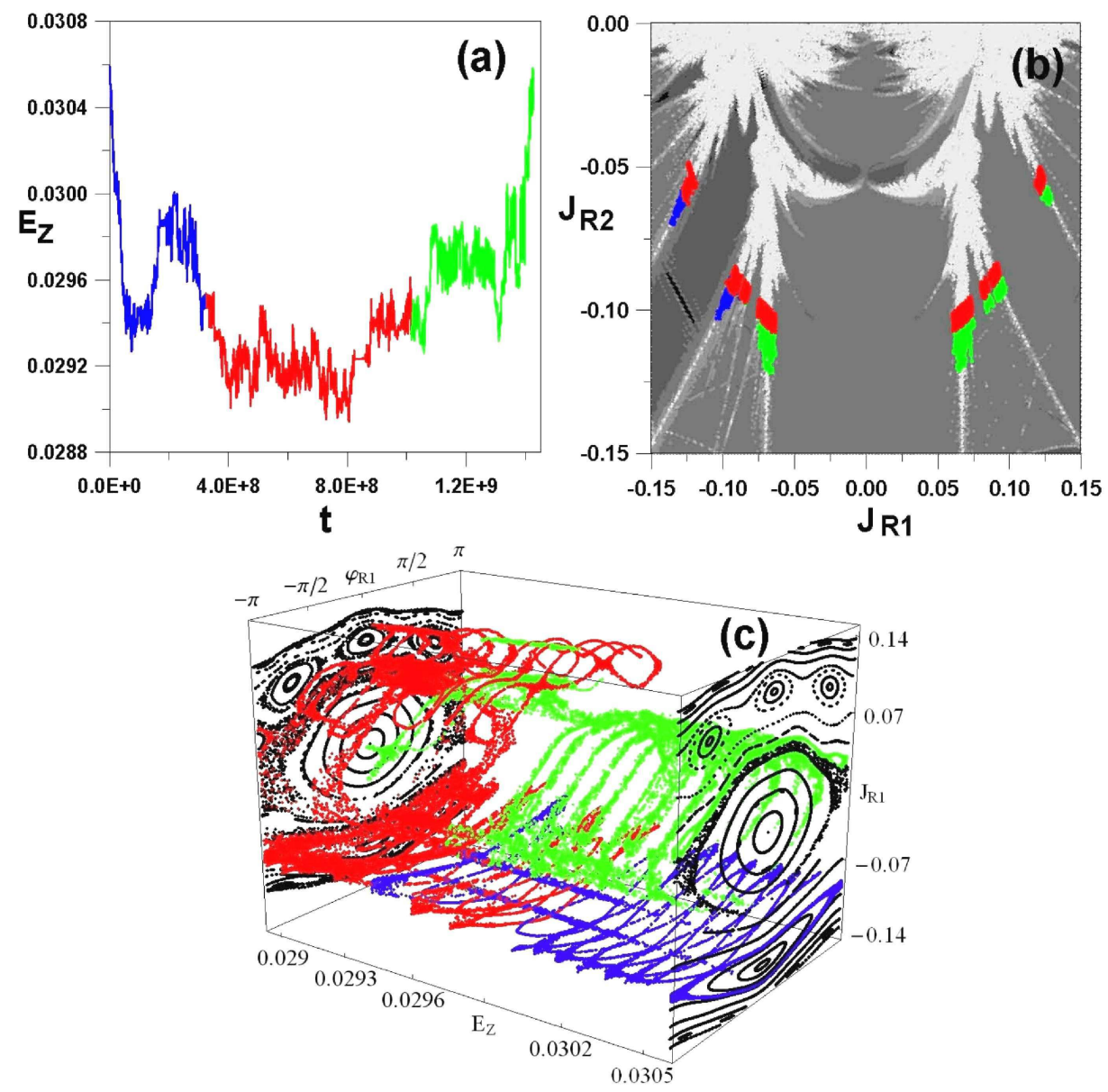

Figure 7: Visualization of Arnold diffusion in appropriate variables of the doubly-resonant normal form, for a numerical orbit in the Hamiltonian (53) for $\epsilon=0.008$. After computing the optimal normal form, we find, via the Lie canonical transformations, the values of all transformed variables $J_{R_{1}}(t), J_{R_{2}}(t), J_{F}(t)$ and $\phi_{R_{1}}(t), \phi_{R_{2}}(t), \phi_{F}(t)$ corresponding to particular values of the old variables $J_{1}(t), J_{2}(t), J_{3}(t)$ and $\phi_{1}(t), \phi_{2}(t), \phi_{3}(t)$ stored at many different times $t$ within an interval $0 \leq t \leq 1.5 \times 10^{9}$ along the numerical run. Using the numerical values of the computed transformed variables, (a) shows the variation of the normal form energy $E_{Z}(t)$ as a function of $t$ in the intervals $0 \leq t \leq 3 \times 10^{8}$ (blue), $3 \times 10^{8} \leq t \leq 10^{9}$ (red), and $10^{9} \leq t \leq 1.5 \times 10^{9}$ (green). The initial and final values are equal to $E_{Z}(t=0)=E_{Z}(t=$ $\left.1.5 \times 10^{9}\right)=0.0306$, while the minimum value, occurring around $t=8 \times 10^{8}$ is $E_{Z}=0.029$. (b) The evolution of the orbit in the action space $\left(J_{R_{1}}, J_{R_{2}}\right)$, using the same colors as in (a) for the corresponding time intervals. In the first time interval (blue), the orbit wanders in the thin chaotic layer of the resonance $\omega_{1}+3 \omega_{2}-\omega_{3}=0$. In the second time interval (red) it jumps first to the resonance $3 \omega_{1}-\omega_{2}-\omega_{3}=0$, and then to the resonance $\omega_{1}-2 \omega_{2}=0$. In the third time interval (green) the orbit recedes from the doubly-resonant domain along the resonance $\omega_{1}-2 \omega_{2}=0$. (c) $3 \mathrm{D}$ plot in the variables $\left(\phi_{R_{1}}, J_{R_{1}}, E_{Z}\right)$, visualizing Arnold diffusion for the same orbit. Taking 20 equidistant values of $E_{Z, i}, i=1,2, \ldots 20$ in the interval $0.029 \leq E_{Z} \leq 0.0306$, we first find the times $t_{i}$ in the interval $0 \leq t \leq 9 \times 10^{8}$ when the normal form energy value $E_{Z}(t)$ of the numerical orbit approaches closest to the values $E_{Z, i}$. For each $i$, starting with the momentary values of all resonant variables at $t_{i}$, we then compute 1000 Poincaré consequents of the normal form flow on the same section as in Figs 66, d. The same procedure is repeated in a second interval $9 \times 10^{8} \leq t \leq 1.5 \times 10^{9}$. As a net result, the orbit at the beginning and end of the calculation is found on the same section (corresponding to $E_{Z}=0.0306$ ), but in a different resonant layer, having by-passed the barriers (invariant tori of the normal form dynamics) via a third dimension (here parameterized by the time-varying value of $E_{Z}$ ). 
In Fig 7, the evolution of the orbit is shown for a total time $t=1.5 \times 10^{9}$. The optimal normal form for $\epsilon=0.008$ has also been computed, whose optimal normalization order is $r_{\text {opt }}=12$, corresponding to an optimal Fourier order $K_{o p t}=36$. Since the corresponding Lie generating functions are known, we compute, via the composition of Lie canonical transformations, the values of all transformed variables $J_{R_{1}}(t), J_{R_{2}}(t), J_{F}(t)$ and $\phi_{R_{1}}(t), \phi_{R_{2}}(t), \phi_{F}(t)$ corresponding to particular values of the old variables $J_{1}(t), J_{2}(t), J_{3}(t)$ and $\phi_{1}(t), \phi_{2}(t), \phi_{3}(t)$ stored at many different times during the numerical run, i.e. as $t$ varies within the interval $0 \leq t \leq 1.5 \times 10^{9}$. Finally, since the exact algebraic expression for the normal form $Z$ is known, we compute the precise numerical value of the normal form energy $E_{Z}(t)$ at the same times.

Fig 7 7 shows the variation of the normal form energy $E_{Z}(t)$ as a function of the time $t$ in the intervals $0 \leq t \leq 3 \times 10^{8}$ (blue), $3 \times 10^{8} \leq t \leq 10^{9}$ (red), and $10^{9} \leq t \leq 1.5 \times 10^{9}$ (green). The final time is such that the initial and final values of $E_{Z}$ are equal, namely $E_{Z}(t=0)=$ $E_{Z}\left(t=1.5 \times 10^{9}\right)=0.0306$. On the other hand, as $E_{Z}$ slowly changes during the run, it acquires a minimum value around $t=8 \times 10^{8}$, which is $E_{Z, \text { min }}=0.029$. Such evolution corresponds to the process described schematically in Fig 1 (section 2). Namely, from the previous figure (Fig 6) we conclude that the two extreme values of $E_{Z}$ acquired during the numerical run are such that $E_{Z}(t=0)>E_{Z c}$ while $E_{Z, \min }<E_{Z c}$, where $E_{Z c}$ is the critical energy corresponding to a large scale overlapping of resonances (subsection 2.3.1). Furthermore, as we will see in the next subsection, the chaotic excursions of the orbits, and, consequently, time evolution of $E_{Z}$, can be approximated by a normal diffusion process. Furthermore, the fastest evolution takes place in the intervals $0 \leq t \leq 10^{8}$, and $1.3 \times 10^{9} \leq t \leq 1.4 \times 10^{9}$, in both of which the total variation of $E_{Z}$ is of the order of $10^{-3}$, or a 'per step' variation of the order of $\Delta E_{Z} \sim 10^{-11}$. It should be stressed that these extremely small variations are possible to unravel numerically only because we use the new canonical variables deduced by the normalizing sequence of Lie canonical transformations. When the old variables are used, instead, we find that the there are large variations (of order $\epsilon^{1 / 2}$ ) of all quantities depending on the actions. These variations are, in fact, dominated by the so-called (in the Nekhoroshev theory) 'deformation' effects (which are also of order $\epsilon^{1 / 2}$ ), hence completely covering the drift effects which are much smaller in size. This feature of the optimal canonical transformations will be exploited in the measurement of the diffusion coefficient $D$ as described in the next subsection.

Fig 7b shows the diffusion of the orbit in the action space $\left(J_{R_{1}}, J_{R_{2}}\right)$, using the same colors as in Fig $7 \mathrm{l}$ for the corresponding time intervals (the background produced by the FLI map is shown here in gray scale). In the first time interval (blue), the orbit wanders chaotically within the thin chaotic layer of the resonance $\omega_{1}+3 \omega_{2}-\omega_{3}=0$. It should be stressed that this wandering has a random walk character, i.e. the orbit makes several reversals of its drift direction, sometimes approaching and other times receding from the center of the double-resonance. On average, however, the drift is in the inward direction (this is a statistical effect; for other initial conditions the average drift turns to be outwards). In the second time interval (red), the orbit jumps first to the domain of the resonance $3 \omega_{1}-\omega_{2}-\omega_{3}=0$. Now, however, the chaotic motion takes place with a relatively high speed (of order $\epsilon^{1 / 2}$ ) in the direction across resonances. As a result, the orbit fills nearly ergodically the whole connected chaotic domain surrounding the main overlapping resonances, while, at the end of this time interval, the orbit is closer to the resonance $\omega_{1}-2 \omega_{2}=0$. Finally, in the third time interval (green) the orbit recedes from the doubly-resonant domain (this is also a statistical effect) being trapped along the domain of the resonance $\omega_{1}-2 \omega_{2}=0$. In this way, at the time $t=1.5 \times 10^{9}$, the orbit is found at about the same distance from the center as initially (at $t=0$ ), but on a different resonance.

Fig 77, now, shows a 3D plot in the variables $\left(\phi_{R_{1}}, J_{R_{1}}, E_{Z}\right)$, visualizing the 'third dimension' 
along which the Arnold diffusion progresses for the same orbit. From this plot we can clearly see the effect of the remainder, which can be considered as a very slow modification of the normal form dynamics acting on a timescale of the order of $10^{9}$ periods. The normal form dynamics, on the other hand, describes well the motion over shorter timescales, of the order of $10^{4}-10^{5}$ periods. In order to show the dynamical effects happening on both timescales, we adopt the following numerical procedure: Taking 20 equidistant values of $E_{Z, i}, i=1,2, \ldots 20$ in the interval $0.029 \leq E_{Z} \leq 0.0306$, we first find the times $t_{i}$ within the interval $0 \leq t \leq 9 \times 10^{8}$ (where the motion is, in general, in the inward direction) when the normal form energy value $E_{Z}(t)$ of the numerical orbit approaches the closest possible to the values $E_{Z, i}$. Then, for each $i$, we set the momentary values of all canonical variables of the numerical orbit at the time $t_{i}$ as initial conditions via which we compute the corresponding values of all the new resonant canonical variables following the composition of the corresponding Lie canonical transformations. With these values as initial conditions, we compute 1000 Poincaré consequents of the normal form flow alone on the same surface of section as defined in Figs 6 , d. The same procedure is repeated in the second interval $9 \times 10^{8} \leq t \leq 1.5 \times 10^{9}$, where the motion is in general in the outward direction. The whole set of Poincaré consequents (points $\left(\phi_{R_{1}}, J_{R_{1}}\right)$ gathered in this way are plotted in the 2D sections of the parallelepiped of Fig $7 \mathrm{k}$, along with the variations of the value of the normal form energy $E_{Z}(t)$ (sampled more frequently) which are shown in the third dimension.

The details of the filling process of the various resonant chaotic layers located in the doublyresonant domain are now clearly seen. In particular, we note that the chaotic orbit fills the whole separatrix layer of the initial resonance $\omega_{1}+3 \omega_{2}-\omega_{3}=0$ in a timescale much shorter than the one required for substantial drift in the $E_{Z}$ direction. After a transient 'back and forth' motion around $E_{z}=0.03$, the orbit then moves slowly towards the value $E_{Z}=0.029$, where all important resonances overlap. In the intermediate time interval (red), we clearly see the filling of the stochastic layers of both resonances $3 \omega_{1}-\omega_{2}-\omega_{3}=0$ and $\omega_{1}-2 \omega_{2}=0$, while global transport is allowed by the normal form dynamics from one resonance to the other. As, however, the remainder effect causes a new motion of the orbit outwards (i.e. towards higher values of $E_{Z}$ (green)), the orbit is eventually captured at the resonance $\omega_{1}-2 \omega_{2}=0$, and stays there until the end of the simulation at $t=1.5 \times 10^{9}$.

It should be emphasized that the fact that the orbit moves in the outward direction at $t=$ $1.5 \times 10^{9}$ does not guarantee that there will be no further return inwards. In fact, we find that most orbits undergo several 'in-out' cycles like the one described in Fig 7 before eventually abandoning the doubly-resonant domain. As an estimate, for $\epsilon=0.008$ we find that the number of cycles before a final exit from the doubly-resonant domain is of the order of 10 , while the total time required for this effect is of the order of $10^{10}$ to $10^{11}$ periods. Furthermore, the probability of exit along one particular resonance decreases as the order of the resonance increases. This is expected, since the width of resonances scales with their order $|k|$ as $\sim e^{-\sigma|k| / 2}$, while the fast filling of the innermost chaotic domains where all the resonances overlap is nearly ergodic.

Finally, we point out that a visualization of the diffusion process like in Fig.7 clearly suggests that the diffusion is driven by the intersections of the asymptotic manifolds of lowerdimensional objects (like hyperbolic 2D tori) all along the path in which the diffusion takes place. However, locating such tori, and studying their manifolds is a task that cannot be accomplished by the use of the Birkhoff normal form as above. on the other hand, the latter provides good initial conditions for a numerical search of such tori. This subject is proposed for future study. 

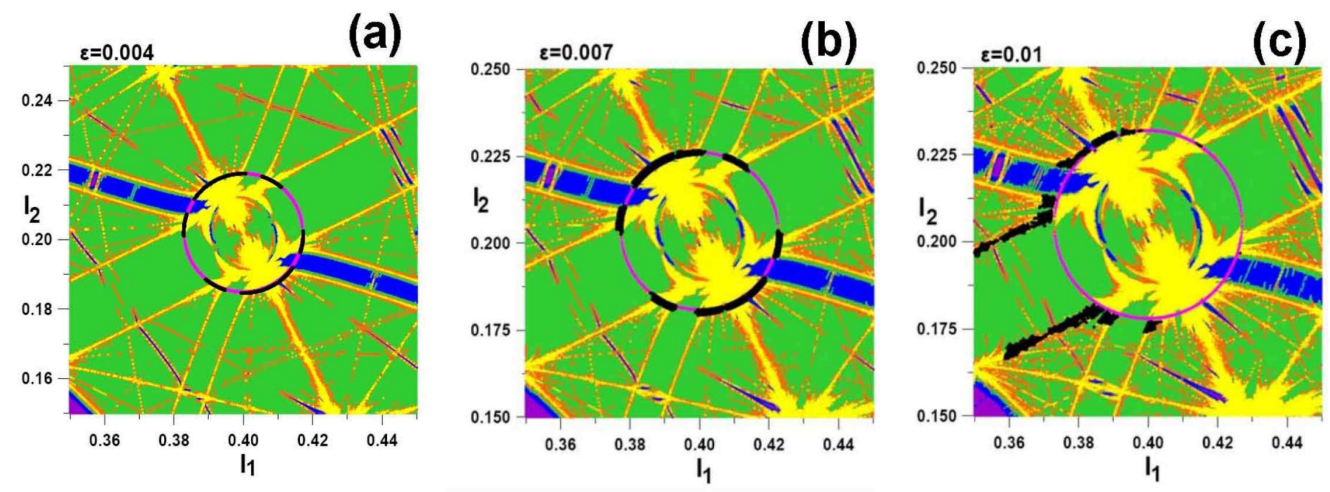

Figure 8: Three orbits with initial conditions on the circle $R(0)=\epsilon^{1 / 2} \rho_{0}$ with $\rho_{0}=0.27$, for (a) $\epsilon=0.004$, (b)) $\epsilon=0.007$ and (c) $\epsilon=0.01$. The black points show the orbits' consequents on the surface of section up to a time $t=10^{8}$. All three orbits are diffusing outwards. The circle with radius $R(0)$ is shown in pink.

\subsection{Dependence of the diffusion coefficient on the optimal remainder}

Our final goal is to obtain numerical estimates of the value of the diffusion coefficient $D$ as well as its relation to the size $\left\|R_{\text {opt }}\right\|$ of the optimal normal form remainder as $\epsilon$ is varied in the interval $0.003 \leq \epsilon \leq 0.020$.

To this end we implement the following numerical procedure: For any fixed value of $\epsilon$, using the information from the FLI maps, we first select 100 initial conditions corresponding to on a circle defined as in Eq. 69, where the radius is chosen equal to

$$
\rho=\rho_{0}=0.27 \text {. }
$$

For such a choice of $\rho$, the corresponding circle lies inside the resonance overlap domain, ensuring that the short time dynamics is dominated by the doubly-resonant normal form. However, in longer times all these orbits exhibit weakly chaotic diffusion. The complete set of initial conditions for one orbit on the circle $\rho=\rho_{0}=0.27$ are found by solving simultaneously for $I_{1}$ and $I_{2}$ the equation of the circle $(\mathrm{Eq} .68 \mathrm{68})$ as well as an equation for the initial angle $\phi_{0}=\arctan \left[\left(I_{2}-0.2\right) /\left(I_{1}-0.4\right)\right]$, where, for each initial condition, $\phi_{0}$ is chosen by visual inspection so as to correspond to an initial condition in the domain of each one of the main overlapping resonances.

We then follow numerically these orbits for a time long enough so that the mean change of their radial distance from the center is large enough to allow for a reliable computation of the diffusion coefficient. Let $R(t)=\epsilon^{1 / 2} \rho(t)$ be the instantaneous value of the distance from the center for any such orbit. The quantity $[R(t)-R(0)]^{2}$ changes as an orbit slowly drifts from one circle to another. Figure 8 shows this effect for three orbits corresponding to the same initial angle $\phi_{0}$ but for three different values of $\epsilon$, namely $\epsilon=0.004$ (Fig $\left.8 \mathrm{~b}\right), \epsilon=0.007$ (Fig $\sqrt[8]{6}$ ), and $\epsilon=0.01$ (Fig 8 c). The orbits are shown by the black points on the section $\left|\phi_{1}\right|+\left|\phi_{2}\right|<0.1$, $\phi_{3}=0$, superposed as usually to the colored background of the FLI map. The pink circles in each panel are the circles $R(0)=\epsilon^{1 / 2} \rho_{0}$, where the orbits' initial conditions lie.

Apart from an overall change of the size of the circle of initial conditions with $\epsilon$, a simple visual comparison of the three panels suffices to conclude that they imply quite different diffusion rates of their depicted orbits. In all three panels, the orbits (black points) are shown up to a time 

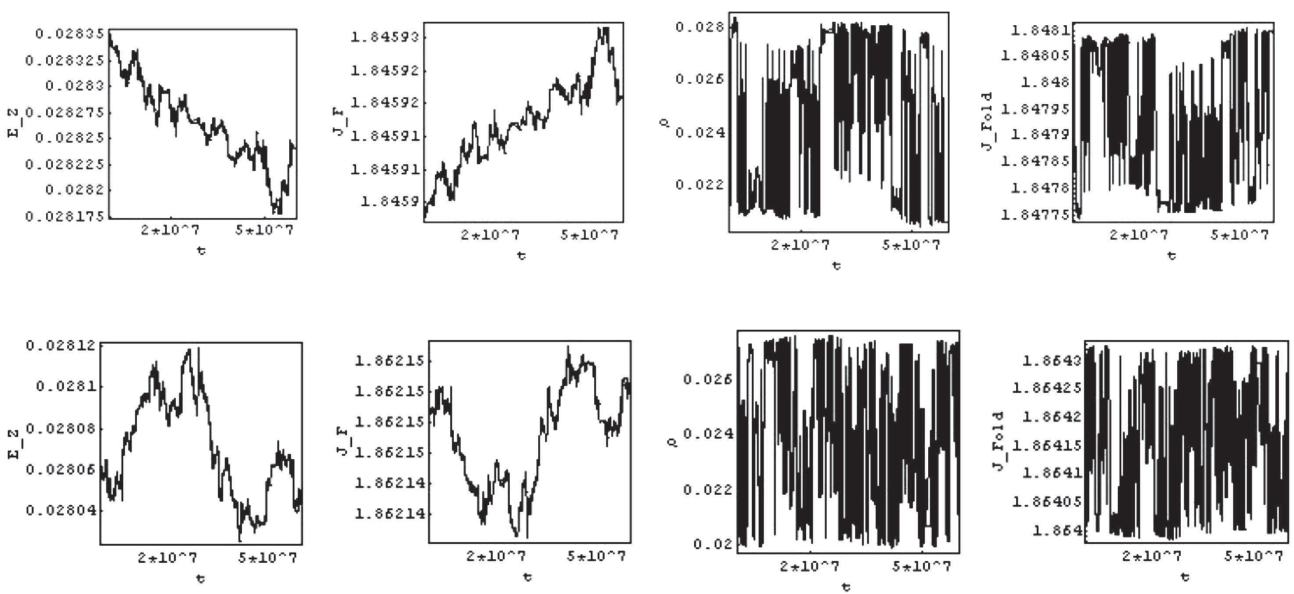

Figure 9: The time evolution of the quantities $E_{Z}$ and $J_{F}$ (see text), using the new transformed canonical variables (first and second panel), and $\rho(t)$ and $J_{F}$ using the original canonical variables (third and fourth panel), for two chaotic orbits of our chosen ensemble for $\epsilon=0.008$ (upper and lower row).

$t=10^{8}$, which is quite long compared to the time needed to fill the chaotic domain along the circle $\rho=\rho_{0}$. However, when $\epsilon=0.004$ (Fig 8 a), the orbit's plot shows that the orbit exhibits no discernible transverse motion with respect to this circle, despite the fact that the orbit lies entirely within a rather strong chaotic domain (yellow in the FLI scale). On the other hand, when $\epsilon$ is raised to $\epsilon=0.007$ (Fig $8 \mathrm{~b}$ ), the orbit is observed to create a small ring around its initial circle, implying that the diffusion is visible in this timescale. Increasing $\epsilon$ still further $(\epsilon=0.01$, Fig $8 \mathrm{r})$, causes now a rather fast diffusion, which leads to the orbit following clearly a preferential 'exit resonance', where the diffusion continues essentially as in the simple resonance case (subsection 2.3.2).

A key remark, now, is the following: similarly to the case of the orbit of Fig 7 whose dynamical features were possible to unravel using the new, i.e., transformed canonical variables after an optimal normalizing transformation, exploiting the same variables, instead of the original ones, allows to observe the random walk-like drift of one orbit in the action space in a much shorter integration time than by the use of the original variables. An example is given in Fig, 9, for $\epsilon=0.008$. We compute, via the optimal normalizing canonical transformation, a time sequence of the values of all the transformed canonical variables $\left(J^{\left(r_{o p t}\right)}(t), \phi^{\left(r_{o p t}\right)}(t)\right)$ from the available sequences of values of the original variables $J(t), \phi(t)$ along the numerical orbits. The four panels in each row show the time evolution, for one chaotic orbit on the circle $\rho_{0}=0.27$, of the quantities i) $E_{Z}$ computed in the transformed canonical variables, ii) $J_{F}=\left(2 J_{1}+J_{2}+5 J_{3}\right) / 30$ computed in the transformed variables, iii) $\rho(t)$ computed in the original canonical variables, and iv) $J_{F}$ computed in the original variables. We note immediately the gain by passing the data through the optimal normalizing transformation, namely the fact that this transformation absorbs all 'deformation' effects, allowing to see the very slow drift due to the weakly chaotic diffusion in a timescale $t \sim 10^{7}$. In fact, the quantity $E_{Z}$ can only be computed in the transformed canonical variables, in which, for both orbits, it undergoes variations of the order $10^{-4}$. In comparison, the analog of $E_{Z}$ in the original variables, i.e., $\rho(t)$, undergoes variations in the second digit, and the 

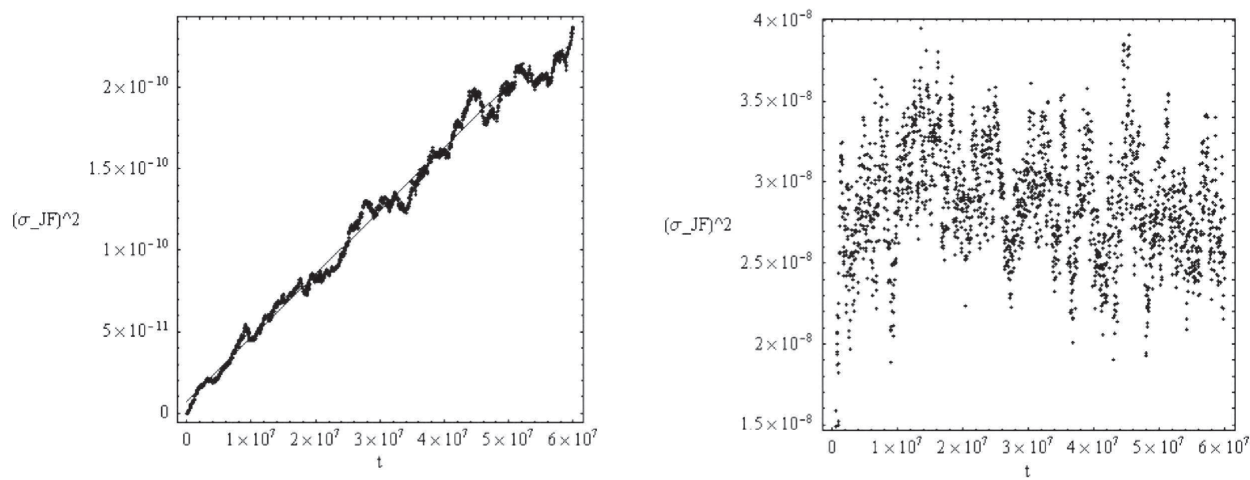

Figure 10: The time evolution of the quantity $\sigma_{J_{F}}^{2}$ (see text), in our ensemble of numerical data for $\epsilon=0.008$, when computed by use of the new transformed canonical variables (left), or the original canonical variables (right). The evolution is shown up to the time $6 \times 10^{7}$.

corresponding time evolution is dominated by $O\left(\epsilon^{1 / 2}\right)$ oscillations, which completely hide the slow drift process in the radial direction with respect to the central doubly resonant point. The comparison is even more straightforward in the variables $J_{F}$ computed by the transformed and by the original action variables. In the former, we can clearly see the drift phenomenon for both orbits, which results in a slow change of the value of $J_{F}$ (which is an approximate integral) at the fifth digit. In contrast, this phenomenon is completely hidden when $J_{F}$ is computed in the original variables, since the corresponding plot is dominated by oscillations of at least one order of magnitude larger amplitude than the drift effect.

In order, now, to measure the value of the diffusion coefficient, using the data from all 100 orbits, we define the mean square deviation:

$$
\sigma_{y}^{2}(t)=\frac{1}{100} \sum_{i=1}^{100}\left(y_{i}(t)-\bar{y}(t)\right)^{2}
$$

where $y(t)=Y(t)-Y(0)$, and $Y(t)$ stands for any of the four quantities shown in Fig 9 . Plotting $\sigma_{y}^{2}$ against the time $t$ allows to estimate the diffusion coefficient. Figure 10 shows an example of this calculation, setting $Y$ equal to $J_{F}$ in the transformed variables (left panel), or the original variables (right panel). We note again that it becomes possible to observe the diffusion in a timescale $t \sim 10^{7}$ using the ensemble of data in the transformed variables, while this time is quite short to reveal any linear trend of $\sigma_{J_{F}}^{2}$ with the time $t$ in the original variables. In fact, in the original variables it was possible to measure reliably the diffusion coefficient only after an integration time $t=10^{9}$. Furthermore, this time increases even more for smaller values of $\epsilon$.

Figure 11 shows the final result. Computing, as indicated above, the diffusion coefficients $D_{E_{Z}}$ and $D_{J_{F}}$ in the transformed canonical variables, for eleven different values of $\epsilon$ as noted in the caption, we also use the data from Fig, 4, whereby we obtain the optimal remainder value $\left\|R_{\text {opt }}\right\|$ for the same values of $\epsilon$ (from the minima of the curves of Fig 4). We then plot $D_{E_{Z}}$ and $D_{J_{F}}$ against $\left\|R_{\text {opt }}\right\|$ in a log-log scale. Despite some scatter, the correlation of both independent 


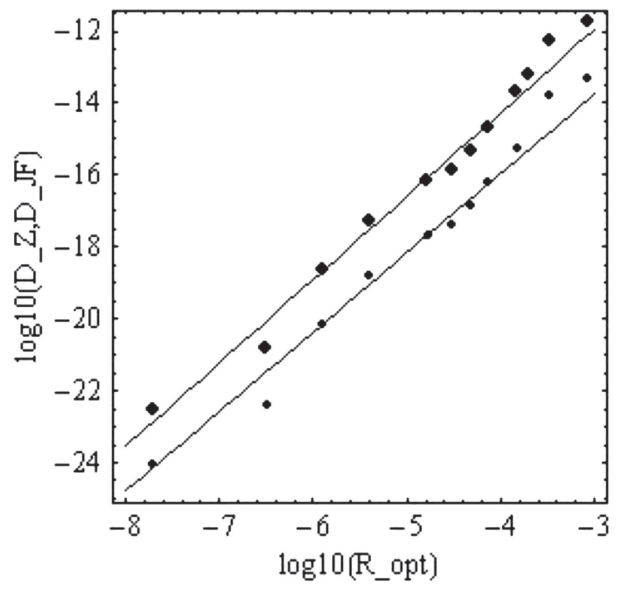

Figure 11: Log-log plot of the dependence of the two estimates of the diffusion coefficient $D_{E_{Z}}$ (upper set of points) and $D_{J_{F}}$ (lower set of points) on the optimal normal form remainder $\left\|R^{\left(r_{o p t}\right)}\right\|$, using numerical data from the integration of orbits (see text). The points correspond to the values of $\epsilon$ (from left to right) $0.003,0.004,0.005,0.007,0.008,0.01$, $0.012,0.013,0.015,0.018,0.020$. The straight lines represent the power-law fits $\log _{10}\left(D_{E_{Z}}\right)=-5+2.3 \log _{10}\left(\left\|R_{o p t}\right\|\right)$ (upper) and $\log _{10}\left(D_{E_{J_{F}}}\right)=-7.1+2.2 \log 10\left(\left\|R_{\text {opt }}\right\|\right)$ (lower).

estimates of the diffusion coefficient with $\left\|R^{\left(r_{o p t}\right)}\right\|$ can be described as a power-law. The powerlaw exponents found by best-fitting are $p=2.3$ for the data of $D_{E_{Z}}$ and $p=2.2$ for the data of $D_{J_{F}}$. In these best fittings we excluded the two points for $\epsilon=0.003$ and $\epsilon=0.004$, since the value of the optimal remainder found by extrapolation is uncertain for these values of $\epsilon$. However, we note that the corresponding points in Fig 11 are still very close to the fitting law found by the remaining data.

The exponents found in Fig 4 are not far from the theoretical estimate $p=2$ derived in section 2 (Eq.45) . However, we have made various trials to determine $p$ via alternative definitions of the diffusion coefficient, and we always find estimations of $p$ somewhat larger than 2 . We thus conjecture that this difference from $p=2$ is a real effect (not due to numerical uncertainties), which, however, requires a more detailed theory to interpret. On the other hand, the corresponding analysis for simple resonances (subsection 2.3.2) as well as the numerical results of [20] indicate that the steepening of the power law in simple resonances of order not smaller than $K^{\prime}$ is quite substantial, leading closer to $p \simeq 3$. In the latter case, another independent example [12] yields $p \approx 2.5$. The issue of how exactly to quantify the steepening of the power-law remains open.

\section{Conclusions}

We examined in detail the phenomenon of weak chaotic diffusion in doubly or simply resonant domains of Hamiltonian systems of three degrees of freedom satisfying the necessary conditions for the holding of the Nekhoroshev theorem. The aim was to determine a quantitative relation between the diffusion coefficient $D$ and the size of the optimal remainder $\left\|R_{o p t}\right\|$ of a resonant normal form constructed according to the requirements of the analytical part of the Nekhoroshev theorem. Our main results are the following: 
1) We propose an efficient algorithm for Hamiltonian normalization, which is implemented as a computer algebraic program performing expansions up to a high order. We explain the practical aspects of this algorithm, and show how it can be used in order to compute i) the optimal normalization order $r_{\text {opt }}$ as a function of the small parameter $\epsilon$, and ii) an estimate of the size of the remainder $\left\|R_{\text {opt }}\right\|$ at the order $r_{\text {opt }}$. The dependence of $r_{\text {opt }}$ on $\epsilon$ is found to be an inverse power-law with an exponent in agreement with theory.

2) We construct estimates on the speed of diffusion in doubly resonant domains. To this end, we examine first the dynamics under the Hamiltonian flow induced by the normal form alone (i.e. neglecting the remainder). The role of the convexity conditions assumed for the original Hamiltonian is analyzed in the context of the normal form dynamics. We then discuss the influence of the remainder on dynamics. Estimates on the value of the diffusion coefficient $D$ are quantified by considering a 'random walk' model for the slow drift of the value of the normal form energy due to the remainder. The final prediction is a power-law estimate $D \sim\left\|R_{\text {opt }}\right\|^{p}$ with $p \simeq 2$ in doubly resonant domains.

3) We perform detailed numerical experiments aiming to test the above predictions, employing the same Hamiltonian model as in [22] as well as the 'FLI map' method. Using the information from the computed normalizing canonical transformations, we propose a convenient set of variables in which the Arnold diffusion in the doubly resonant domains is clearly visualized. Furthermore, using ensembles of chaotic orbits, we make two independent numerical calculations of the diffusion coefficient $D$ for various values of $\epsilon$. The relation between $D$ and $\left\|R_{\text {opt }}\right\|$ found by the two calculations is $D \sim\left\|R_{\text {opt }}\right\|^{2.2}$ and $D \sim\left\|R_{\text {opt }}\right\|^{2.3}$ respectively.

4) Finally, we make some theoretical estimates on the relation between $D$ and $\left\|R_{\text {opt }}\right\|$ in simply resonant domains. In this case, we combine the basic theory developed in [9] together with estimates given in [49] regarding the dependence of the size of the separatrix splitting on the optimal normal form remainder in simply resonant domains. We are thus led to the prediction $\left\|R_{\text {opt }}\right\|^{2(1+b)}$, where $b \simeq 1 / 2$, or $p=2(1+b) \simeq 3$, holding for all simple resonances of order higher than $K^{\prime}$, where $K^{\prime}$ is defined in Eq.(12). The latter result interprets the results obtained in an earlier study [20] by purely numerical means.

Acknowledgements: We thank two anonymous referees for a thorough revision of our manuscript, with many constructive suggestions, as well as Prof. G. Contopoulos for careful reading of the manuscript. C.E. acknowledges fruitful discussions with the group of C. Froeschlé, M. Guzzo and E. Lega.

\section{References}

[1] Arnold, V.I., 1963: Russ. Math. Surveys 18, 9.

[2] Arnold, V.I., 1964: Sov. Math. Dokl. 6, 581.

[3] Benettin, G., Galgani, L., and Giorgilli, A.: 1985, Cel. Mech. 37, 1.

[4] Benettin, G., and Gallavotti, G.: 1986, J. Stat. Phys. 44, 293.

[5] Benettin G., Fassò F., Guzzo M.: 1998, Regular Chaot. Dyn., 3, 56.

[6] Benettin, G.: 1999, in A. Giorgilli (Ed.) 'Hamiltonian Dynamics. Theory and Applications'. Lect. Notes Math. 1861, 1.

[7] Cachucho, F., Cincotta, P.M., and Ferraz-Mello, S.: 2010, Cel. Mech. Dyn. Astron. 108, 35.

[8] Celletti, A., and Ferrara, L.: 1996,Cel. Mech. Dyn. Astron. 64, 261.

[9] Chirikov, B.V.: 1979, Phys. Rep. 52, 263.

[10] Chirikov, B.V., and Vecheslavov, V.V.: 1985, Physica 71, 243.

[11] Cincotta, P.: 2002, New Astronomy Reviews 46, 13.

[12] Cincotta, P., Giordano, C., Mestre, M., and Efthymiopoulos, C: 2013, in preparation. 
[13] Contopoulos, G.: 1966, Bull. Astron. CNRS 2, 223.

[14] Contopoulos, G.: 2002, Order and Chaos in Dynamical Astronomy, Springer, Berlin.

[15] Dumas, H.S., and Laskar, J.: 1993, Phys. Rev. Lett. 70, 2975.

[16] Efthymiopoulos, C., Contopoulos, G. and Voglis, N.: 1998, in Benest, D., and Froeschlé, C. (Eds) Discrete Dynamical Systems, Gordon and Breach Science Publishers, pp.91-106.

[17] Efthymiopoulos, C., Giorgilli, A., and Contopoulos, G: 2004, J. Phys. A Math. Gen. 37, 10831.

[18] Efthymiopoulos, C.: 2005, Cel. Mech. Dyn. Astron. 92, 29.

[19] Efthymiopoulos, C., and Sandor, Z.: 2005, Mon. Not. R. Astron. Soc. 364, 253.

[20] Efthymiopoulos, C.: 2008, Cel. Mech. Dyn. Astron. 102, 49.

[21] Ferraz-Mello, S.: 2007, Canonical Perturbation Theories. Degenerate Systems and Resonance. Springer (New York).

[22] Froeschlé, C., Guzzo, M., and Lega, E.: 2000, Science 289 (5487), 2108.

[23] Froeschlé, C., Guzzo, M., and Lega, E.: 2005, Cel. Mech. Dyn. Astron. 92, 243.

[24] Gelfreich, V., Simó, C., and Vieiro, A.: 2013, Physica D 243, 92.

[25] Giordano, C.M., and Cincotta, P.M.: 2004, Astron. Astrophys. 423, 745.

[26] Giorgilli A., Delshams A., Fontich E., Galgani L., Simó C.: 1989, J. Differ. Eq., 77, 167.

[27] Giorgilli A., and Locatelli, U.: 1997, ZAMP, 48, 220.

[28] Giorgilli A., and Skokos, C.: 1997, Astron. Astrophys., 317, 254.

[29] Giorgilli, A.: 1999, In: C. Simo (ed.), Hamiltonian Systems with Three or More Degrees of Freedom, Kluwer, Dordrecht.

[30] Giorgilli, A: 2002, Notes on exponential stability of Hamiltonian systems, in Dynamical Systems. Part I: Hamiltonian Systems and Celestial Mechanics, Pubblicazioni della Classe di Scienze, Scuola Normale Superiore, Pisa.

[31] Giorgilli, A., Locatelli, U., and Sansoterra, M.: 2009, Cel. Mech. Dyn. Astron. 104, 159.

[32] Guzzo, M., Lega, E., and Froeschlé, C.: 2002, Physica D 163, 1.

[33] Guzzo, M., Lega, E., and Froeschlé, C.: 2005, Dis. Con. Dyn. Sys. B 5, 687.

[34] Guzzo, M., Lega, E., and Froeschlé, C.: 2006, Nonlinearity 19, 1049.

[35] Guzzo, M., Lega, E., and Froeschlé, C.: 2011, Chaos 21, 033101.

[36] Kaneko, K., and Konishi, T.: 1989, Phys. Rev. A 40, 6130.

[37] Laskar, J.: 1993, Physica D67, 257.

[38] Lega, E., Guzzo, M., and Froeschlé, C.: 2003, Physica D 182, 179.

[39] Lega, E., Froeschlé, C., and Guzzo, M.: 2007, Lect. Notes Phys. 729, 29.

[40] Lega, E., Guzzo, M., and Froeschlé, C.: 2009, Cel. Mech. Dyn. Astron. 104, 191.

[41] Lega, E., Guzzo, M., and Froeschlé, C.: 2010a, Cel. Mech. Dyn. Astron. 107, 129.

[42] Lega, E., Guzzo, M., and Froeschlé, C.: 2010b, Cel. Mech. Dyn. Astron. 107, 115.

[43] Lhotka, Ch., Efthymiopoulos, C., and Dvorak, R.: 2008, Mon. Not. R. Astron. Soc. 384, 1165

[44] Liechtenberg, A.J., and Lieberman, M.A.: 1992, Regular and Chaotic Dynamics, Springer, Berlin.

[45] Lochak, P.: 1992, Russ. Math. Surv. 47, 57

[46] Mather, J.: 2004, J. Math. Sci. 124, 5275.

[47] Morbidelli, A.: 2002, Modern Celestial Mechanics. Aspects of Solar System Dynamics, Taylor and Francis, London.

[48] Morbidelli, A., and Guzzo, M.: 1997, Cel. Mech. Dyn. Astron. 65, 107.

[49] Morbidelli, A., and Giorgilli, A.: 1997, Physica D 102, 195.

[50] Neishtadt, A.I.: 1984, J. Appl. Math. Mech. 48, 133.

[51] Nekhoroshev, N.N.: 1977, Russ. Math. Surv. 32(6), 1.

[52] Pavlovic, R., and Guzzo, M.: 2008, Mon. Not. R. Astron. Soc. 384, 1575.

[53] Poincaré, H: 1892, Méthodes Nouvelles de la Mécanique Céleste, Gautier-Vilard, Paris.

[54] Pöshel, J.: 1993, Math. Z. 213, 187.

[55] Rosenbluth, M., Sagdeev, R., Taylor, J., and Zaslavskii, M.: 1966, Nucl. Fusion 6, 217.

[56] Simó, C., and Valls, C.: 2001, Nonlinearity 14, 1707.

[57] Tennyson, J.: 1982, Physica D 5, 123

[58] Skokos, C., Contopoulos, G., and Polymilis, C.: 1997, Cel. Mech. Dyn. Astr. 65, 223.

[59] Wood, B.P., Lichtenberg, A.J., and Lieberman, M.A.: 1990,Phys. Rev. A 42, 5885.

\section{Appendix A. Quasi-convexity and normal form energy constraints}

The quadratic form $\zeta_{0,2}$ given by Eq. 29) can be written as:

$$
\zeta_{0,2}=\left(J_{R_{1}}, J_{R_{2}}\right) \cdot k^{(1,2)} \cdot M_{*} \cdot\left(k^{(1,2)}\right)^{T} \cdot\left(J_{R_{1}}, J_{R_{2}}\right)^{T}
$$


where $k^{(1,2)}$ is a $2 \times 3$ matrix whose first and second line are given by $\left(k_{1}^{(1)}, k_{2}^{(1)}, k_{3}^{(1)}\right)$ and $\left(k_{1}^{(2)}, k_{2}^{(2)}, k_{3}^{(2)}\right)$ respectively. Since the matrix $M_{*}$ is real symmetric, it can be writen in the form $M_{*}=X \cdot \mu_{*} \cdot X^{T}$, where $\mu_{*}=\operatorname{diag}\left(\mu_{1}, \mu_{2}, \mu_{3}\right)$, with $\mu_{i}=$ the eigenvalues of $M_{*}$, while $X$ is an orthogonal matrix with columns equal to the normalized eigenvectors of $M_{*}$. Using the above expression for $M_{*}$, Eq. A.1 resumes the form

$$
\zeta_{0,2}=\left(J_{R_{1}}, J_{R_{2}}\right) \cdot Y \cdot \mu_{*} \cdot Y^{T}\left(J_{R_{1}}, J_{R_{2}}\right)^{T}
$$

where $Y=k^{(1,2)} \cdot X$ is a $2 \times 3$ matrix. Writing $\zeta_{0,2}$ as $\zeta_{0,2}=Q J_{R_{1}}^{2}+V J_{R_{1}} J_{R_{2}}+P J_{R_{2}}^{2}$, and denoting by $y_{i j}$ the elements of $Y$, the discriminant $\Delta=4 Q P-V^{2}$ is given by:

$$
\Delta=-\left[\left(y_{11} y_{22}-y_{12} y_{21}\right)^{2} \mu_{1} \mu_{2}+\left(y_{11} y_{23}-y_{13} y_{21}\right)^{2} \mu_{1} \mu_{3}+\left(y_{12} y_{23}-y_{13} y_{22}\right)^{2} \mu_{2} \mu_{3}\right]
$$

Since we have assumed (subsection 2.1) that either all three eigenvalues $\mu_{i}$ have the same sign, or two of them have the same sign and one is zero, by Eq. A.2 we have that $\Delta<0$. That is, the quadratic form $\zeta_{0,2}$ is positive definite. 